\title{
EXTENDED MODULAR OPERAD
}

\author{
A. Losev ${ }^{1}$, Yu. Manin ${ }^{2}$ \\ ${ }^{1}$ Institute of Theoretical and Experimental Physics, Moscow, Russia \\ ${ }^{2}$ Max-Planck-Institut für Mathematik, Bonn, Germany \\ and Northwestern University, Evanston, USA
}

\begin{abstract}
This paper is a sequel to [LoMa] where moduli spaces of painted stable curves were introduced and studied. We define the extended modular operad of genus zero, algebras over this operad, and study the formal differential geometric structures related to these algebras: pencils of flat connections and Frobenius manifolds without metric. We focus here on the combinatorial aspects of the picture. Algebraic geometric aspects are treated in [Ma2].
\end{abstract}

\section{$\S 0$. Introduction and summary}

This paper, together with [Ma2], constitutes a sequel to [LoMa] where some new moduli spaces of pointed curves were introduced and studied. We start with a review of the main results of [LoMa] and then give a summary of this paper.

0.1. Painted stable curves. Let $S$ be a finite set. A painting of $S$ is a partition of $S$ into two disjoint subsets: white $W$ and black $B$.

Let $T$ be a scheme, $S$ a painted set, $g \geq 0$. An $S$-pointed (or labeled) curve of genus $g$ over $T$ consists of the data

$$
\left(\pi: C \rightarrow T ; x_{i}: T \rightarrow C, i \in S\right)
$$

where

(i) $\pi$ is a flat proper morphism whose geometric fibres $C_{t}$ are reduced and connected curves, with at most ordinary double points as singularities, and $g=$ $H^{1}\left(C_{t}, \mathcal{O}_{C_{t}}\right)$.

(ii) $x_{i}, i \in S$, are sections of $\pi$ not containing singular points of geometric fibres.

(iii) $x_{i} \cap x_{j}=\emptyset$ for all $i \in W, j \in S, i \neq j$.

Such a curve (0.1) is called painted stable, if the normalization of any irreducible component $C^{\prime}$ of a geometric fibre carries $\geq 3$ pairwise different special points when $C^{\prime}$ is of genus 0 and $\geq 1$ special points when $C^{\prime}$ is of genus 1 . Special points are inverse images of singular points and of the structure sections $x_{i}$. Equivalently, such a normalization has only a finite automorphism group fixing the special points.

We get the usual notion of a (family of) stable painted curves, if $S$ consists of only white points. 
The (dual) modular graph of a geometric fibre is defined in the same way as in the usual case (for the conventions we use see [Ma1], III.2). Tails now can be of two types, we may refer to them and their marks as "black" and "white" ones as well, and call the graph painted one. More on the geometry of such graphs and morphisms between them see in the subsections 1.1-1.2 below. The isomorphism class of a painted modular graph is called the combinatorial type of the respective curve.

0.2. Moduli stacks $\bar{L}_{g, S}$. In sec. 4 of [LoMa], we gave two equivalent descriptions of the stacks $\bar{L}_{g, S}$ where $S$ is a painted set. In [Ma2], Lemma 1.2.2, a third description was given based upon a more general definition due to B. Hassett $[\mathrm{H}]$. For reader's convenience, we will reproduce them here.

(i) $\bar{L}_{g, S}$ consists of families of curves of chain stable combinatorial types.

To define the latter, consider first combinatorial types of classical (semi)stable curves with only white points labeled by a finite set $W$. They are isomorphism classes of graphs, whose vertices are labeled by "genera" $g \geq 0$ and tails are bijectively labeled by elements of $W$. Stability means that vertices of genus 0 bound $\geq 3$ flags, and vertices of genus 1 bound $\geq 1$ flags. Graphs can have edges with only one vertex, that is, simple loops.

Starting with the geometric realization of such a graph $\Gamma$, we can obtain an infinite series of painted graphs, which will be called chain stable. Namely, subdivide edges and tails of $\Gamma$ by a finite set of new vertices of genus zero (on each edge or tail, this set may be empty). If a tail was subdivided, move the respective label (from $W$ ) to the newly emerged tail. Distribute the black tails labeled by elements of $B$ arbitrarily among the old and the new vertices. The resulting graph is called chain stable if it becomes stable after repainting black tails into white ones. The new vertices depict chains of $\mathbf{P}^{1}$ 's stabilized by black points. Each end of such a chain carries either a white tail or a singular special point (or both).

(ii) The second description of $\bar{L}_{g, S}$ is based upon the inductive construction of "adjoining a new black point".

Namely, starting with an $S$-pointed family $\left(C / T,\left(x_{i} \mid i \in S\right)\right)$ as in (0.1) we will produce another $S^{\prime}$-pointed family $\left(C^{\prime} / T^{\prime},\left(x_{i}^{\prime} \mid i \in S^{\prime}\right)\right)$ where $T^{\prime}=C, S^{\prime}=S \cup\{*\}$, * being a new black label. The new curve and sections will be produced in two steps. At the first step we make in (0.1) the base change $C \rightarrow T$. We get an $S$-pointed curve $X:=C \times{ }_{S} C$, with sections $x_{i, C}$. We then add an extra section $\Delta: C \rightarrow C \times{ }_{S} C$ which is the relative diagonal, and mark it by $*$. We have not yet produced an $S^{\prime}$-pointed curve over $T^{\prime}=C$, because the extra black section will intersect singular points of the fibres and white sections as well.

Now comes the second step of the construction, where we birationally modify $C \times_{T} C \rightarrow C$ as in $[\mathrm{Kn}]$, Definition 2.3. Define $C^{\prime}:=\operatorname{Proj} \operatorname{Sym} \mathcal{K}$ as the relative 
projective spectrum of the symmetric algebra of the sheaf $\mathcal{K}$ on $X=C \times_{T} C$ which is defined as the cokernel of the map

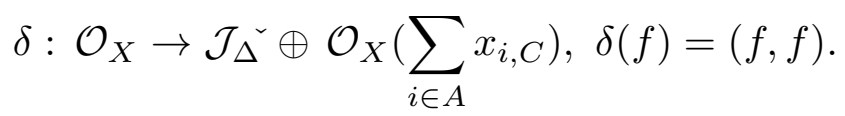

Here $\mathcal{J}_{\Delta}$ is the $\mathcal{O}_{X}$-ideal of $\Delta$, and $\mathcal{J}_{\Delta}$ is its dual sheaf considered as a subsheaf of meromorphic functions, as in [Kn], Lemma 2.2 and Appendix.

As a result, we get an $S^{\prime}$-pointed curve, because Knudsen's treatment of his modification is local and can be directly extended to our case. If the initial curve was painted stable, the new one will be painted stable as well.

Moreover, this construction is obviously functorial, in the sense that it produces a stack if we started with a stack.

We are now ready to construct $\bar{L}_{g, S}$. For concreteness, we assume that $W=$ $\{1, \ldots, m\}$ and $B=\{1, \ldots, n\}$ are initial segments of (two copies of) natural numbers.

If $g \geq 2, m \geq 0$, we start with $\bar{M}_{g ; m}=\bar{M}_{g ; m, \emptyset}$ and adjoin $n$ new black points, one in turn. Denote the resulting stack by $\bar{L}_{g ; m, n}$.

For $g=1$, we repeat this construction for $m \geq 1$. We need one more sequence of stacks, corresponding to $m=0$ (elliptic curves stabilized only with black points). Since we want to restrict ourselves to Deligne-Mumford stacks, we start with $\bar{M}_{1 ; 0,1}$ which is defined by repainting the white point in $\bar{M}_{1 ; 1}$, and adjoin black points to get the sequence $\bar{L}_{1 ; 0, n}, n \geq 1$.

Finally, for $g=0$ we obtain the series of spaces $\bar{L}_{n}=\bar{L}_{0 ; 2, n}$, thoroughly studied in [LoMa] and moreover $\bar{L}_{0 ; m, n}$, for all $m \geq 3, n \geq 0$.

From our construction it follows that the genus zero stacks are actually smooth manifolds.

(iii) The third, and technically most useful, description identifies $\bar{L}_{g, S}$ with certain stacks of weighted pointed stable curves studied in [H], see also [Ma2], Definition 1.2.1. Roughly speaking, in Hassett's definition we endow each section $x_{s}$ with a weight $a_{s} \in \mathbf{Q} \cap(0,1]$. Weighted stability means that (an appropriate power of) $\omega_{C / T}\left(\sum_{s} a_{s} x_{s}\right)$ is relatively ample, and moreover, the sum of weights of any subfamily of $x_{s}$ coinciding on a geometric fiber does not exceed 1. Lemma 1.2.1 in [Ma2] shows that if $S$ is painted, and if we choose weights so that $a_{s}=1$ for $s \in W$ and $\sum_{t \in B} a_{t} \leq 1$, the two definitions of stability coincide.

0.3. Extended modular operad. The classical modular spaces $\bar{M}_{g, m+1}$ come together with the action of $\mathbf{S}_{m+1}$. Moreover, there is a family of clutching morphisms

$$
\bar{M}_{g_{1}, m_{1}+1} \times \bar{M}_{g_{2}, m_{2}+1} \rightarrow \bar{M}_{g_{1}+g_{2}, m_{1}+m_{2}+1}
$$


which on the level of geometric points can be described as gluing one (say, the last) labeled point of the first pointed curve to, say, the first labeled point of the second curve. Finally, there is a similar family of morphisms

$$
\bar{M}_{g-1, m+1} \rightarrow \bar{M}_{g, m-1}
$$

gluing together two different labeled points.

We can axiomatize the evident relations between these structures and get the abstract notion of the modular operad in the category of $D M$-stacks. One can also add a linear structure, by passing to the (homology) motives or any of their realizations and get the notion of the modular operad in a tensor category, as well as the basic example of this notion. This was done by E. Getzler and M. Kapranov in [GeK2]. Restricting ourselves to the $g=0$ case, we get almost a classical operad, with two modifications: first, its $m$-th component $\bar{M}_{g, m+1}$ is acted upon by $\mathbf{S}_{m+1}$ rather than by $\mathbf{S}_{m}$; second, it lacks the $m=1$-component since $\mathbf{P}^{1}$ with only two labeled points is unstable.

The first modification means that we actually deal with a cyclic operad, the notion which was as well introduced and studied by E. Getzler and M. Kapranov ([GeK1]). Recall that generally classical linear operads classify $k$-algebras of a given type, like associative, commutative, Lie, Poisson etc. The component $P(m)$ of an operad $P$ (in our case $H_{*}\left(\bar{M}_{0, m+1}\right)$ ) is the linear space of polylinear operations of $m$ arguments that can be constructed from the basic operations (multiplications, commutators etc), modulo identical relations supplied by the axioms (associativity, Jacobi etc). The action of $\mathbf{S}_{m}$ upon $P(m)$ corresponds to the permutations of the arguments. If $A$ is an algebra over $P$, each $p \in P(m)$ defines thus a tensor $p: A^{\otimes m} \rightarrow A$, and we know how these tensors transform under all permutations of $m$ lower indices. If $A$ is endowed with a scalar product, we can lower the $m+1$-th index as well identifying $p$ with a map $A^{m+1} \rightarrow k$. It makes sense to require the scalar product and the action of $\mathbf{S}_{m+1}$ on such tensors to be a part of the structure of algebras of a given type. Cyclic operads then are well-suited for axiomatizing such a situation.

We now pass to the problem of the 1 -component. It is missing because a projective line with two labeled (white) points has an infinite automorphism group $G_{m}$ and so is unstable. The trick suggested in [LoMa] consists in stabilizing $\mathbf{P}^{1}$ with an arbitrary number of additional black points. This forces us to add degenerations which are arbitrary chains of such $\mathbf{P}^{1}$ 's. Thus our candidate for the missing $\bar{M}_{0,2}$ is $\bigsqcup_{n \geq 1} \bar{L}_{0,2, n}$. The remaining spaces $\bar{L}_{g, m, n}$ constitute a minimal extension of the family $\bar{M}_{g, m}$ containing all $\bar{L}_{0,2, n}$ and stable with respect to the operadic (clutching) morphisms.

Specifically, we put

$$
\widetilde{L}_{g, m}:=\coprod_{n} \bar{L}_{g, m+1, n}
$$


Morphisms (0.4) and (0.5) in which we allow to glue together only white points induce the clutching maps

$$
\begin{aligned}
\widetilde{L}_{g_{1}, m_{1}} \times \widetilde{L}_{g_{2}, m_{2}} & \rightarrow \widetilde{L}_{g_{1}+g_{2}, m_{1}+m_{2}}, \\
\widetilde{L}_{g-1, m+1} & \rightarrow \widetilde{L}_{g, m-1} .
\end{aligned}
$$

The symmetry group $\mathbf{S}_{m+1}$ acts upon $\widetilde{L}_{g, m}$ by renumbering white points.

Moreover, each stack $\widetilde{L}_{g, m}$ is a union of infinitely many irreducible $D M$-stacks which are indexed by the number of black points; the group $\mathbf{S}_{n}$ acts on the $n$-th component by renumbering black points. These structures induce the respective symmetries of the linearized versions. This is the price we paid for acquiring the $m=1$ component. It has a nice meaning in the language of the respective algebras: an algebra now consists of two spaces $(A, T)$; polylinear operations are of the form $A^{\otimes m} \otimes T^{\otimes n} \rightarrow A$. They depend on $t_{i} \in T$ as parameters which are associated with black points. Thus we acquired degrees of freedom allowing us to encompass certain deformations.

The key result of the theory of the (cyclic) homology operad $\left\{H_{*}\left(\bar{M}_{0, m+1}\right)\right\}$ consists in establishing a bijection between finite-dimensional operadic algebras and formal Frobenius manifolds. A more detailed analysis which we relegate to a future paper shows that in our present context we get exactly the deformations associated with gravitational descendants.

0.4. Genus zero extended operad. It is convenient to imagine its bigraded components $\bar{L}_{0, m+1, n}$ sitting at the lattice points $(m, n)$ of the first quadrant. Then the lowest horizontal line is the classical genus zero modular operad $\bar{M}_{0, m+1}$ whereas the leftmost vertical line represents the 1-component of the extended operad. Operadic multiplication must induce a monoid structure on the 1-component, and in our case it is supplied by the appropriate clutching morphisms described above and more formally in [Ma2], [LoMa]:

$$
\widetilde{L}_{0, m_{1}+1} \times \widetilde{L}_{0, m_{2}+1} \rightarrow \widetilde{L}_{0, m_{1}+m_{2}+1}
$$

In $[\mathrm{LoMa}]$, it was proved that the appropriately defined algebras over the 1component $\widetilde{L}_{0,1}$ bijectively correspond to the pencils of formal flat connections. The treatment in [LoMa] stressed also the toric picture of $\widetilde{L}_{0,1}$; for certain generalizations of this picture, cf. [R].

Thus at the boundary of the $(m, n)$-quadrant we have a pretty detailed understanding of the geometry of the respective moduli spaces, algebraic structure of their homology and cohomology including the operadic formalism, and finally, a 
reduction of the theory of operadic algebras to the study of specific differential equations.

The main goal of [Ma2] and this paper taken together is to extend these results from the boundary of the $(m, n)$-quadrant to the whole quadrant.

Here we focus mainly on the combinatorial aspects of the picture; those arguments for which algebraic geometry is indispensable, are treated in [Ma2].

0.5. Contents of the paper. In $\S 1$ and $\S 2$ we define for any painted set $S$ purely combinatorially a graded ring $H_{S}^{*}$ and a graded module $H_{* S}$ over this ring and study their structure. Using the results of this study and additional geometric arguments, we establish in [Ma2] canonical isomorphisms $H_{S}^{*}=H^{*}\left(\bar{L}_{0 S}\right)$ and $H_{* S}=H_{*}\left(\bar{L}_{0 S}\right)$, together with identification of the action of $H_{S}^{*}$ upon $H_{* S}$ as the cap product.

In $\S 3$ we extend the construction of $H^{*}$ and $H_{*}$ to the labeled trees replacing $S$. Algebraic geometric meaning of this extension consists in studying (co)homology of moduli spaces of painted stable curves of a given combinatorial type and their degenerations. Combinatorially, these rings/modules together with various morphisms connecting them constitute a version of graded (co)operad.

In $\S 4$ we introduce several versions of algebras over this (co)operad and describe them in terms of correlators and top correlators.

Finally, in $\S 5$ we demonstrate the geometric meaning of the differential equations satisfied by the generating series for top correlators. There are two basic geometric languages, corresponding to what we call in $\S 5$ the commutativity and the oriented associativity equations. One language leads to the study of pencils of flat connections as in [LoMa1], another to a version of "Frobenius manifolds with affine flat structure but without invariant metric". Both versions are related by the induction of flat affine structure from a fiber. This procedure first appeared in [Lo1],[Lo2].

Acknoledgements. The research of A. L. was partially supported by grants RFBR 01-01-00548, by INTAS-99-590 and by support of scientific schools grant 00-1596557.

\section{$\S 1$. Combinatorial cohomology rings}

1.1. Graphs. We define a graph $\tau$ as a quadruple $\left(V_{\tau}, F_{\tau}, \partial_{\tau}, j_{\tau}\right)$ where $V_{\tau}$, resp. $F_{\tau}$, are finite sets of vertices, resp. flags; $\partial_{\tau}: F_{\tau} \rightarrow V_{\tau}$ is the boundary, or incidence, map; $j_{\tau}: F_{\tau} \rightarrow F_{\tau}$ is an involution of the set of flags. This combinatorial definition is related to the more common one via the notion of the geometric realization of $\tau$. The latter is a topological space which is obtained from $F_{\tau}$ copies of $[0,1]$ by gluing together points 0 in the copies corresponding to each vertex $v \in V_{\tau}$, and by

gluing together points 1 in each orbit of $j_{\tau}$. This motivates the introduction of the following auxiliary sets and their geometric realizations: the set $E_{\tau}$ of edges of $\tau$, 
formally consisting of cardinality two orbits of $j_{\tau}$, and the set $T_{\tau}$ of tails, consisting of those flags, which are $j_{\tau}$-invariant.

We will very often think and speak about graphs directly in terms of their geometric realizations. In particular, $\tau$ will be called connected (resp. tree, resp. forest) if its geometric realization is connected (resp. connected and has no loops, resp. is a disjoint union of trees).

Situations in which one must be more careful and invoke the initial combinatorial definition usually occur when one has to consider morphisms of graphs. We will define here three types of generating morphisms which we will need. For more sophisticated definitions, cf. [BeMa].

In fact, $[\mathrm{BeMa}]$ considers graphs endowed with additional labeling of their vertices with integers $\geq 0$. Such graphs arise as dual graphs of (semi)stable pointed curves: vertices correspond to the irreducible components, labels to the genera of their normalizations. This part of the structure can be neglected in most of this paper which treats only the combinatorics of the genus zero curves whose dual graphs are trees with all vertices labeled by zero.

Moreover, flags are in a bijection with the special points of the normalized curve. Hence when $S$ is weighted and/or painted, we must consider graphs whose flags are weighted, resp. painted.

(i) An isomorphism $\sigma \rightarrow \tau$ consists of two bijections $\varphi^{F}: F_{\tau} \rightarrow F_{\sigma}, \varphi_{V}: V_{\sigma} \rightarrow$ $V_{\tau}$. They must be compatible with $\partial_{\sigma}, \partial_{\tau}$, and $j_{\sigma}, j_{\tau}$. As a corollary, they induce bijections of edges and tails as well. (Notice that maps between flags go in reverse direction with respect to the morphisms of graphs. This convention will persist below.)

(ii) Let $\sigma$ be a graph, and $e$ its edge consisting of two flags $e_{1}, e_{2}$. A morphism $c: \sigma \rightarrow \tau$ contracting $e$ geometrically simply deletes the interior of $e$ if it is a loop with vertex $v$, or else contracts $e$ so that its ends $v_{1}, v_{2}$ become a new vertex $v$ otherwise. Combinatorially, it is given by a map $c^{F}: F_{\tau} \rightarrow F_{\sigma}$ which identifies $F_{\tau}$ with $F_{\sigma} \backslash\left\{e_{1}, e_{2}\right\}$ and is compatible with involutions, and a map $c_{V}: V_{\sigma} \rightarrow V_{\tau}$ which glues together vertices of $e_{1}, e_{2}$ and is a bijection elsewhere. Distribution of flags between all vertices except for those of $e_{1}, e_{2}$ must be compatible with these maps. Flags of $\tau$ at $v$ (if $e$ is not a loop) are thus $F_{\sigma}\left(v_{1}\right) \cup F_{\sigma}\left(v_{2}\right) \backslash\left\{e_{1}, e_{2}\right\}$.

(iii) Let $\sigma$ be a graph and $\left\{f_{1}, f_{2}\right\}$ its two different tails. A morphism $\sigma \rightarrow \tau$ which glues these two tails together consists of two bijections $F_{\tau} \rightarrow F_{\sigma}, V_{\sigma} \rightarrow V_{\tau}$ compatible with $\partial_{\sigma}, \partial_{\tau}$, and making of $\left\{f_{1}, f_{2}\right\}$ one new edge.

Composition of morphisms is the composition of the relevant maps (in reverse order for flags). A composition of contractions of edges and isomorphisms will be called simply a contraction. The category of trees and contractions will reappear in $\S 3$. 
1.1.1. Painted graphs and labeled graphs. A painting of $\tau$ is a partition of its tails into two subsets: white and black. If a painting is not given explicitly, we assume that all tails are white.

Morphisms between painted graphs are subject to the following restrictions: isomorphisms and contractions do not change color of tails; it is allowed to glue together only white tails.

Let $S$ be a set, and $\tau$ a graph. An $S$-marking (or labeling) of $\tau$ is a bijection $S \rightarrow T_{\tau}$. Equivalently, we call a graph with an $S$-marking an $S$-graph, and a morphism between two $S$-graphs identical on $S$, an $S$-morphism. We identify a painting of $\tau$ with a painting of $S$.

1.1.2. Stable and stably painted trees. Let now $\tau$ be a tree. We recall that it is called stable, iff its every vertex is incident to at least three flags.

A painted tree is called painted stable, iff

(i) It is stable.

(ii) Every end vertex of $\tau$ carries at least one white tail. (An end vertex is a vertex incident to 1 or 0 edges).

In particular, an one edge $S$-tree is the same as an unordered 2-partition $S=$ $\sigma_{1} \cup \sigma_{2}$ showing how the tails are distributed between the two vertices. Painted stability means that $\sigma_{1}$ and $\sigma_{2}$ each contain at least one white point, and $\left|\sigma_{i}\right| \geq 2$.

We will often use the following notation. If $S_{1}, S_{2}$ are two subsets of $S$ and $\sigma$ is a 2-partition of $S$, then $S_{1} \sigma S_{2}$ is a shorthand for the following statement: all elements of $S_{1}$ lie in one part of $\sigma$ whereas all elements of $S_{2}$ lie in another one. If one of the sets is empty, we may omit it.

1.2. 2-partitions and trees. Let $\tau$ be a tree, $e$ its edge. If we delete the interior of (the geometric realization of) $e$, the tree will split into two connected components, and the set of its tails will break into two subsets. If $\tau$ is $S$-marked, we get thus an unordered 2-partition $\sigma_{e}$ of $S$. If $\tau$ is painted stable, this partition will also be painted stable.

Let $\sigma=\left(\sigma_{1}, \sigma_{2}\right)$ and $\tau=\left(\tau_{1}, \tau_{2}\right)$ be two non-trivial unordered partitions of $S$. Denote by $\delta(\sigma, \tau)$ the number of non-empty pairwise distinct sets among $\sigma_{k} \cap \tau_{l}$ diminished by 2 .

Clearly, $\delta(\sigma, \tau)$ can take only values $0,1,2$. Moreover,

(i) $\delta(\sigma, \tau)=0$ iff $\sigma=\tau$.

(ii) $\delta(\sigma, \tau)=1$ iff the following condition is satisfied. There exists a partition of $S$ into three (non-empty pairwise disjoint) subsets $\rho_{i}, i=1,2,3$, such that one of the partitions $\sigma, \tau$ is $\left(\rho_{1} \cup \rho_{2}, \rho_{3}\right)$, and another is $\left(\rho_{1}, \rho_{2} \cup \rho_{3}\right)$. The partition $\left(\rho_{i}\right)$ is determined uniquely up to a permutation of $\rho_{1}$ and $\rho_{3}$. 
To see this, notice that there must be exactly one empty intersection $\sigma_{a} \cap \tau_{b}$. Renumbering the sets if necessary we may assume that $\sigma_{2} \cap \tau_{1}=\emptyset$. Then $\rho_{1}:=$ $\tau_{1}, \rho_{2}:=\sigma_{1} \cap \tau_{2}$ and $\rho_{3}:=\sigma_{2}$ will do.

Moreover, assume in this situation that $\sigma$ and $\tau$ are painted stable as one-edge trees. Denote by $\sigma * \tau$ the two-edge $S$-tree whose tails are distributed between its three vertices according to the partition $\left(\rho_{i}\right)$, with $\rho_{2}$ in the middle. Then $\sigma * \tau$ is stable painted as well. In fact, its end vertices carry at least two tails of which at least one is white because this holds for $\sigma$ and $\tau$; and its middle vertex carries at least three flags because $\rho_{2}$ is non-empty.

(iii) $\delta(\sigma, \tau)=2$ iff there exists an ordered quadruple of pairwise distinct elements $i, j, k, l \in S$ such that jiokl and $j k \tau i l$. Moreover, if $S$ is painted and $\sigma, \tau$ are painted stable, $i, j, k, l$ can be chosen in such a way that the partitions induced by $\sigma$ and $\tau$ on this quadruple are also painted stable.

In fact, if one disregards stability, it suffices to choose $j \in \sigma_{1} \cap \tau_{1}, i \in \sigma_{1} \cap \tau_{2}$, $k \in \sigma_{2} \cap \tau_{1}, l \in \sigma_{2} \cap \tau_{2}$. This is possible exactly when $\delta(\sigma, \tau)=2$.

If $\sigma$ and $\tau$ are painted stable, consider the $(2,2)$ matrix of sets $\left(\sigma_{a} \cap \tau_{b}\right)$. A contemplation will convince the reader that at least one of the two diagonals of this matrix has the following property: each of its elements contains a white label. Hence either $i$ and $k$, or else $j$ and $l$ can be chosen white. In both cases, the induced partitions of $\{i, j, k, l\}$ are painted stable.

We will call a set $E$ of pairwise distinct (painted) stable 2-partitions $\{\sigma\}$ of a (painted) stable set $S$ good, if $\delta\left(\sigma, \sigma^{\prime}\right)=1$ for any $\sigma \neq \sigma^{\prime}$ in $E$. In particular, empty set is good, and any one-element set is good.

1.2.1. Lemma. (a) For any (painted) stable $S$-tree $\tau$, the set $\left\{\sigma_{e} \mid e \in E_{\tau}\right\}$ is good.

(b) The map $\tau \mapsto\left\{\sigma_{e} \mid e \in E_{\tau}\right\}$ establishes a bijection between (painted) stable $S$-trees up to an $S$-isomorphism, and good families.

(c) Let $E^{\prime}$ be a non-empty good family, $\sigma \in E^{\prime}, E=E^{\prime} \backslash\{\sigma\}$. Let $\tau^{\prime}$, resp. $\tau$, be the respective $S$-trees, and $e \in E_{\tau^{\prime}}$ the edge corresponding to $\sigma$. Then there is a unique $S$-morphism $\tau^{\prime} \rightarrow \tau$ contracting e and inducing the tautological embedding $E \subset E^{\prime}$.

Proof. If one disregards painting, statements (a) and (b) are proved in the Proposition 3.5.2 of Chapter III of [Ma1]. To deal with painted trees, it remains only to check that if one starts with a non-empty good family and produces the respective tree, it will be painted stable, that is, its every end vertex carries a white tail. In fact, such a vertex belongs to a unique edge $e$, and the tails carried by it constitute a part of the partition $\sigma_{e}$. Since $\sigma_{e}$ is painted stable, the conclusion follows. 
We leave (c) as an exercise to the reader.

1.2.2. Proposition. Let $\tau$ be a painted stable $S$-tree, and $\sigma$ a painted stable 2-partition of $S$. Then exactly one of the following alternatives takes place.

(i) There exists an edge $e \in E_{\tau}$ such that $\sigma=\sigma_{e}$. In this case we will say that $\sigma$ breaks $\tau$ at $e$.

(ii) There exists a vertex $v \in V_{\tau}$, an $S$-tree $\tau^{\prime}$ and an $S$-morphism $\tau^{\prime} \rightarrow \tau$ contracting an edge e of $\tau^{\prime}$ to the vertex $v$ of $\tau$ such that $\sigma=\sigma_{e}$. In this case we will say that $\sigma$ breaks $\tau$ at $v$.

(iii) None of the above. In this case there is an edge $e \in E_{\tau}$ such that $\delta\left(\sigma, \sigma_{e}\right)=2$. We will say that $\sigma$ does not break $\tau$.

Proof. Consider the family of numbers $\delta\left(\sigma, \sigma_{e}\right), e \in E_{\tau}$. If it contains zero, we are in the case (i), and then all other numbers are 1, because of Lemma 1.2.1 (a). If it consists only of 1's, we can add $\sigma$ to $\left\{\sigma_{e}\right\}$ and get a good family, which produces a new tree $\tau^{\prime}$ and a contracting morphism $\tau^{\prime} \rightarrow \tau$ with required properties. If it contains 2 , we are in the case (iii).

1.2.3. Remark. In the following it will be useful to keep in mind a more detailed picture of various possibilities that can arise in the case (ii).

If we delete a vertex $v$ from the geometric realization of $\tau$, it will break into a set of $\geq 3$ connected components which we will call branches of $\tau$ at $v$. Their set is canonically bijective to the set of flags $F_{\tau}(v)$ incident to $v$ : we can say that the branch starts with the respective flag. In the extreme case, a branch can be a single tail.

Each branch $b$ carries its full subset of tails, or their labels, $S_{b}$, so that all $S_{b}$ together form a partition of $S$. A 2-partition $\sigma$ of $S$ breaks $\tau$ at $v$ precisely when each of its parts is a union of several $S_{b}$ 's. In this way we establish a bijection between such 2-partitions $\sigma$ of $S$ and 2-partitions $\alpha$ of $F_{\tau}(v)$. We will say that $\alpha$ is (painted) stable if the respective $\sigma$ is. Most of $\alpha$ 's will be automatically painted stable. Unstable $\alpha$ 's are of two types:

(i) one part of $\alpha$ consists of one white tail; or

(ii) one part of $\alpha$ consists of only black tails.

In fact, if a branch starts with an edge, then at an end vertex of this edge there are at least two tails, of which at least one is white.

1.3. Combinatorial cohomology. In the following we fix a painted set $S$ with $|S| \geq 3$ containing at least two white elements, and a commutative coefficient ring $k$. Consider the family of independent commuting variables $\left\{l_{\sigma}\right\}$ indexed by painted stable unordered 2-partitions $\sigma$ of $S$ and put $\mathcal{R}_{S}:=k\left[l_{\sigma}\right]$. 
Call an ordered quadruple of pairwise distinct elements $i, j, k, l \in S$ allowed, if both partitions $i j \mid k l$ and $k j \mid i l$ are painted stable. For a painted stable $\sigma$ put $\epsilon(\sigma ; i, j, k, l)=1$ if $\{i, j, k, l\}$ is allowed and $i j \sigma k l ;-1$, if $\{i, j, k, l\}$ is allowed and $k j \sigma i l$; and 0 otherwise.

Define

$$
R_{i j k l}:=\sum_{\sigma} \epsilon(\sigma ; i, j, k, l) l_{\sigma} \in \mathcal{R}_{S}
$$

$R_{\sigma \sigma^{\prime}}:=l_{\sigma} l_{\sigma^{\prime}}$ if there exists an allowed quadruple $i, j, k, l$ with ijokl, $k j \sigma^{\prime} i l$.

Here $\sigma, \sigma^{\prime}$ run over 2 -partitions. Denote by $I_{S} \subset \mathcal{R}_{S}$ the ideal generated by all elements (1.1) and (1.2) and define the combinatorial cohomology ring by

$$
H_{S}^{*}:=\mathcal{R}_{S} / I_{S}
$$

The main result of [Ma2], Theorem 3.1.1, establishes a canonical isomorphism of $H_{S}^{*}$ with the cohomology and the Chow ring of $\bar{L}_{0, S}$. The proof heavily uses calculations of this paper and the Keel's theorem who proved this result in [Ke] for the basic case when there are no black points.

Returning to our combinatorial setup, let now $\tau$ be a painted stable $S$-tree. Put

$$
m(\tau):=\prod_{e \in E_{\tau}} l_{\sigma_{e}} \in \mathcal{R}_{S}
$$

and call such a monomial good. It depends only on the $S$-isomorphism class of $\tau$. If $\tau$ is one-vertex tree, we put $m(\tau)=1$.

1.3.1. Multiplication table. Below we will calculate $l_{\sigma} m(\tau) \bmod I_{S}$. The answer will depend on the mutual position of $\sigma$ and $\tau$ as described in Prop. 1.2.2 which we will consider in reverse order.

Case (iii): $\sigma$ does not break $\tau$. Then

$$
l_{\sigma} m(\tau) \equiv 0 \bmod I_{S}
$$

because in this case $l_{\sigma} m(\tau)$ is divisible by one of the elements (1.2).

Case (ii): $\sigma$ breaks $\tau$ at a vertex $v$. Then

$$
l_{\sigma} m(\tau)=m(\sigma * \tau)
$$

where $\sigma * \tau$ is the painted stable $S$-tree corresponding to the set of 2 -partitions $\left\{\sigma_{e} \mid e \in E_{\tau}\right\} \cup\{\sigma\}$. 
Case (i): $\sigma$ breaks $\tau$ at an edge $e$. In this case we will write several formulas depending on additional choices. Namely, denote by $v_{1}, v_{2}$ two vertices of $e$ and by $e_{1}, e_{2}$ the respective flags ("halves of $e$ "). Choose two distinct flags $I, J \in$ $F_{\tau}\left(v_{1}\right) \backslash\left\{e_{1}\right\}$ and similarly $K, L \in F_{\tau}\left(v_{2}\right) \backslash\left\{e_{2}\right\}$ with the following property:

$\left(^{*}\right)$ there exist labels $i, j, k, l$ on the branches starting with $I, J, K, L$ respectively, forming an allowed quadruple.

Again, we will call such $I, J, K, L$ an allowed quadruple. It is important to notice that allowed $I, J, K, L$ always exist: it suffices to take for $J$ any branch at $v_{1}$ carrying a white tail, for $L$ any branch at $v_{2}$ carrying a white tail, and complement them by arbitrary $I, K$.

Now we state that for each allowed quadruple of flags as above we have:

$$
l_{\sigma} m(\tau) \equiv-\sum_{\alpha: I J \alpha e_{1}} m(\tau(\alpha))-\sum_{\beta: e_{2} \beta K L} m(\tau(\beta)) \bmod I_{S}
$$

Here $\alpha$ (resp. $\beta)$ runs over painted stable $2-$ partitions of $F_{\tau}\left(v_{1}\right)\left(\operatorname{resp} . F_{\tau}\left(v_{2}\right)\right)$ in the sense of 1.2.3. If $\alpha$ determines a 2-partition $\sigma$ of $S$, we denote by $\tau(\alpha)$ the former $\sigma * \tau$.

To prove (1.7), we choose $i, j, k, l$ as in $\left(^{*}\right)$ and write first of all

$$
R_{i j k l} m(\tau)=\sum_{\rho} \epsilon(\rho ; i, j, k, l) l_{\rho} m(\tau) \in I_{S}
$$

where we sum over 2-partitions $\rho$ of $S$. Now, if $\epsilon(\rho ; i, j, k, l)=-1$, then $k j \rho i l$, and since $i j \sigma k l$, we have $l_{\rho} l_{\sigma} \in I_{S}$; but $l_{\sigma}$ divides $m(\tau)$, and so $l_{\sigma} m(\tau) \in I_{S}$. Hence in the r.h.s. of (1.8) we may sum only over $\rho$ 's with $i j \rho k l$, and $\sigma$ is among this set. Therefore,

$$
l_{\sigma} m(\tau) \equiv-\sum_{\substack{\rho: i j \rho k l \\ \rho \neq \sigma}} l_{\rho} m(\tau) \bmod I_{S}
$$

In the sum (1.9) we may and will omit $\rho$ 's that do not break $\tau$. Moreover, no $\rho$ can break $\tau$ at an edge, or at a vertex distinct from $v_{1}$ or $v_{2}$ : otherwise the condition ij $\rho k l$ cannot hold. Finally, $\rho$ 's which break $\tau$ at $v_{1}$ or $v_{2}$ will produce precisely the right hand side of (1.7).

1.3.2. Corollary. The ring $\mathcal{R}_{S}$ is linearly spanned by $I_{S}$ and good monomials $m(\tau)$. The cohomology ring $H_{S}^{*}$ is spanned by the classes of $m(\tau)$.

In fact, the span of $I_{S}$ and $m(\tau)$ contains 1 and is stable with respect to multiplications by all $l_{\sigma}$. 
However, the space of linear relations $I_{S} \cap\langle m(\tau)\rangle$ is generally non-trivial. Below we will exhibit some elements of this space which we will call the standard ones and which will later be shown to span all the relations.

1.4. Standard relations between good monomials. As above, we will calculate some expressions $R_{i j k l} m(\tau)$. However, formerly we started with a tree $\tau$, an edge $e=e_{\sigma}$ and flags $I, J$ and $K, L$ at two ends of this edge.

Now we will start with a tree $\tau$, a vertex $v$, an allowed quadruple of flags $I, J, K, L$ incident to this vertex, and an allowed quadruple of labels $i, j, k, l$ carried by the respective branches. Looking at the summands at the right hand side of (1.8), we see that if $\rho$ does not break $\tau$, we can omit it, and if $\rho$ breaks $\tau$ at an edge or at a vertex different from $v$, then $\epsilon(\rho ; i, j, k, l)=0$.

Partitions $\rho$ breaking $\tau$ at $v$ correspond to 2-partitions $\alpha$ of $F_{\tau}(v)$. So finally we get the standard relation

$$
\sum_{\alpha} \epsilon(\alpha ; I, J, K, L) m(\tau(\alpha)) \equiv 0 \bmod I_{S}
$$

where the sign $\epsilon$ is \pm 1 depending on whether $I J \alpha K L$ or $K J \alpha I L$.

\section{$\S 2$. Combinatorial homology modules}

2.1. Homology space. We keep notations of $\S 1$, in particular, fix a painted set $S$ and a coefficient ring $k$. Define a free $k$-module $\mathcal{M}_{S}:=\oplus_{\tau} k \mu(\tau)$ freely generated by all $S$-isomorphism classes of painted stable $S$-trees $\tau$. Denote by $J_{S} \subset \mathcal{M}_{S}$ the submodule spanned by the standard relations (1.10) in which $m(\tau)$ are replaced by $\mu(\tau)$ :

$$
r(\tau, v ; I, J, K, L):=\sum_{\alpha} \epsilon(\alpha ; I, J, K, L) \mu(\tau(\alpha)) \in J_{S}
$$

Finally, define the homology $k$-space as

$$
H_{* S}:=\mathcal{M}_{S} / J_{S}
$$

and put $[\mu(\tau)]:=\mu(\tau) \bmod J_{S}$.

The main content of this section is the definition of an action of $H_{S}^{*}$ on $H_{* S}$ which turns the homology module into a free module of rank one over the cohomology ring. To be more precise, we start with defining an action of the ring generators $l_{\sigma}$ upon $\mathcal{M}_{S}$ which imitates the multiplication table (1.5)-(1.7), and proceed to show that it is compatible with the defining relations.

2.2. Multiplication table revisited. We will first formally define the action of $l_{\sigma}$ upon generators $\mu(\tau)$ sending them to some elements of $\mathcal{M}_{S}$. If $\sigma$ does not break $\tau$, we put

$$
l_{\sigma} \mu(\tau)=0 .
$$


If $\sigma$ breaks $\tau$ at a vertex $v$, we put

$$
l_{\sigma} \mu(\tau)=\mu(\sigma * \tau)
$$

Finally, if $\sigma$ breaks $\tau$ at an edge $e$, we choose an arbitrary allowed quadruple of flags $I, J, K, L$ as in 1.3.1, Case (i), and put in the notations explained above

$$
l_{\sigma} \mu(\tau)=-\sum_{\alpha: I J \alpha e_{1}} \mu(\tau(\alpha))-\sum_{\beta: e_{2} \beta K L} \mu(\tau(\beta)) .
$$

2.3. Theorem. Formulas (2.3)-(2.5) induce a well defined action of $H_{S}^{*}$ upon $H_{* S}$.

2.3.1. Corollary. (a) This action makes of $H_{* S}$ a free module of rank one over $H_{S}^{*}$.

(b) The standard relations (1.10) span all linear relations between the classes of good monomials $m(\tau)$ in $H_{S}^{*}$.

Deduction of the Corollary. Since the relations between the classes of $\mu(\tau)$ correspond to a part of linear relations between good monomials, we have a surjective $k$-linear map

$$
s: H_{* S} \rightarrow H_{S}^{*}:[\mu(\tau)] \mapsto[m(\tau)] .
$$

Here we set $[m(\tau)]:=m(\tau) \bmod I_{S}$.

On the other hand, denoting by $1 \in H_{* S}$ the class of one-vertex $S$-tree, thanks to Theorem 2.3 we have another linear map

$$
t: H_{S}^{*} \rightarrow H *_{S}:[m(\tau)] \mapsto[m(\tau)] \mathbf{1}
$$

Induction on the number of edges of $\tau$ using (2.4) shows that $[m(\tau)] \mathbf{1}=[\mu(\tau)]$. Therefore $s$ and $t$ are mutually inverse, so that $\mathbf{1}$ is a free generator of $H *_{S}$ as an $H_{S}^{*}$-module. This completes the deduction.

We now turn to the proof of the theorem which is given in the subsections 2.3.22.3.6. It consists of a chain of sometimes tedious checks, and the reader may prefer to skip it.

2.3.2. Prescription (2.5) is well defined. The right hand side of (2.5) formally depends on the choice of an allowed quadruple $I, J, K, L$. One can pass from one allowed quadruple to another one replacing each time only one flag so that all intermediate quadruples will also be allowed (at each vertex, start with replacing a flag carrying a white label by another flag also carrying a white label). 
We will now take the difference of the right hand sides of (2.5) written for quadruples $I, J, K, L$ and $I^{\prime}, J, K, L$. The second sums will cancel. In the first sums, the terms corresponding to $\alpha$ 's with $I I^{\prime} J \alpha e_{1}$ will also cancel. The remaining terms are

$$
-\sum_{\alpha: I J \alpha I^{\prime} e_{1}} \mu(\tau(\alpha))+\sum_{\alpha: I^{\prime} J \alpha I e_{1}} \mu(\tau(\alpha)) \bmod J_{S}
$$

Clearly, (2.6) is an element of the form (2.1), namely $r\left(\tau, v_{1} ; I^{\prime}, J, I, e_{1}\right)$.

One can similarly treat a replacement of $J$ by $J^{\prime}$.

2.3.3. Multiplication by $l_{\sigma}$ maps $J_{S}$ into itself. As in (2.1), choose a relation $r(\tau, v ; I, J, K, L)$ and a 2 -partition $\sigma$. We want to show that $l_{\sigma} r(\tau, v ; I, J, K, L)$ belongs to $J_{S}$. Consider in turn the following subcases.

(i) $\sigma$ does not break $\tau$

If $\sigma$ does not break $\tau$, it does not break any of $\tau(\alpha)$, hence $l_{\sigma} r(\tau, v ; I, J, K, L)=0$ in view of $(2.3)$.

(ii) $\sigma$ breaks $\tau$ at a vertex different from $v$

In this case we obviously have

$$
l_{\sigma} r(\tau, v ; I, J, K, L)=r(\sigma * \tau, v ; I, J, K, L)
$$

(iii) $\sigma$ breaks $\tau$ at an edge not incident to $v$

In this case we calculate $l_{\sigma} \tau(\alpha)$ using one of the formulas (2.5) which involve surgery only at the ends of $e$ so that it does not interact with the partitions $\alpha$ in (2.1). Hence we get a sum of the elements of the type (2.1) written for various $\tau(\alpha)$.

The remaining two cases are more difficult.

(iv) $\sigma$ breaks $\tau$ at an edge e incident to $v$

We will start with some notation. Let $v_{0}$ be the vertex of $e$ distinct from $v$. Denote by $e_{0}$, resp. $e_{1}$, the flags of $e$ at $v_{0}$, resp. $v$. For each $\alpha$ appearing in (2.1), the tree $\tau(\alpha)$ has an extra edge which we denote $f=f_{\alpha}$; other edges, in particular, $e$, "are" the edges of $\tau$. Clearly, $e$ and $f_{\alpha}$ have in $\tau(\alpha)$ a common vertex $v$.

In order to calculate $l_{\sigma} \mu(\tau(\alpha))$ we have to use a formula of the type (2.5) written for the edge $e$ of $\tau(\alpha)$. Notation in (2.5) conflicts with that in (2.1), so we will rewrite (2.5) as:

$$
l_{\sigma} \mu(\tau(\alpha))=-\sum_{\beta: I_{\alpha} J_{\alpha} \beta e_{0}} \mu(\tau(\alpha)(\beta))-\sum_{\gamma: e_{1} \gamma K_{\alpha} L_{\alpha}} \mu(\tau(\alpha)(\gamma))
$$


Here $I_{\alpha}, J_{\alpha}, K_{\alpha}, L_{\alpha}$ can be chosen depending on $\alpha$, thanks to 2.3.2. Recall from 1.3.1 that these flags must form an allowed quadruple, and

$$
I_{\alpha}, J_{\alpha} \in F_{\tau(\alpha)}\left(v_{0}\right)=F_{\tau}\left(v_{0}\right), \quad K_{\alpha}, L_{\alpha} \in F_{\tau(\alpha)}(v)
$$

Moreover, $\beta$, resp. $\gamma$, run over 2-partitions of $F_{\tau(\alpha)}\left(v_{0}\right)=F_{\tau}\left(v_{0}\right)$, resp. of $F_{\tau(\alpha)}(v)$, whereas $\alpha$ runs over partitions of $F_{\tau}(v)$.

With this notation, we have

$$
\begin{gathered}
l_{\sigma} r(\tau, v ; I, J, K, L)=\sum_{\alpha} \epsilon(\alpha ; I, J, K, L) l_{\sigma} \mu(\tau(\alpha))= \\
-\sum_{\alpha} \epsilon(\alpha ; I, J, K, L)\left(\sum_{\beta: I_{\alpha} J_{\alpha} \beta e_{0}} \mu(\tau(\alpha)(\beta))+\sum_{\gamma: e_{1} \gamma K_{\alpha} L_{\alpha}} \mu(\tau(\alpha)(\gamma))\right) .
\end{gathered}
$$

Now, in (2.9) $\alpha$ and $\beta$ occur at different vertices of $\tau$. Hence $\mu(\tau(\alpha)(\beta))=$ $\mu(\tau(\beta)(\alpha))$, and we can interchange summation on $\alpha$ and $\beta$ if we choose $I_{\alpha}, J_{\alpha}$ independent of $\alpha$. This is possible: namely, choose as $J_{\alpha}=J_{0}$ an arbitrary flag in $F_{\tau}\left(v_{0}\right) \backslash\left\{e_{1}\right\}$ whose branch carries a white label, and for $I_{\alpha}=I_{0}$ choose any other flag in this set. The condition on $J_{0}$ will make a quadruple $I_{0}, J_{0}, K_{\alpha}, L_{\alpha}$ allowed if $L_{\alpha}$ carries a white label as well. We will care about it later.

Meanwhile we can rewrite the first half of (2.9):

$$
-\sum_{\beta: I_{0} J_{0} \beta e_{0}} \sum_{\alpha} \epsilon(\alpha ; I, J, K, L) \mu(\tau(\beta)(\alpha))=-\sum_{\beta: I_{0} J_{0} \beta e_{0}} r(\tau(\beta), v ; I, J, K, L) \in J_{S}
$$

Hence it remains to show that, after an appropriate choice of $K_{\alpha}, L_{\alpha}$,

$$
\text { (?) } \sum_{\alpha} \epsilon(\alpha ; I, J, K, L) \sum_{\gamma: e_{1} \gamma K_{\alpha} L_{\alpha}} \mu(\tau(\alpha)(\gamma)) \in J_{S}
$$

Choice of $K_{\alpha}, L_{\alpha}$. For $L_{\alpha}$ we will always choose the flag of the new edge $f_{\alpha}$ incident to $v$. This choice assures that $I_{0}, J_{0}, K_{\alpha}, L_{\alpha}$ will form an allowed quadruple.

The choice of $K_{\alpha}$ will additionally depend on the mutual position of $e$ and of flags $I, J, K, L$. Notice that in our calculation this set of data is fixed: it determines the left hand side of (2.9).

There are five logical possibilities: one or none of the flags $I, J, K, L$ coincides with $e_{v}$, the flag of $e$ incident to $v$. By symmetry, it suffices to consider three options: none is $e_{v}, I=e_{v}$, or $J=e_{v}$. We need only look at those $\alpha$ for which $I J \alpha K L$ or $K J \alpha I L$. 
Here is the list of choices.

If none of $I, J, K, L$ is $e_{v}$, we put $K_{\alpha}=I$ for $\alpha I e_{v}$ and $K_{\alpha}=K$ for $\alpha K e_{v}$.

If $I=e_{v}$, we put $K_{\alpha}=J$ for $\alpha J e_{v}$ and $K_{\alpha}=L$ for $\alpha L e_{v}$.

Finally, if $J=e_{v}$, we again put $K_{\alpha}=I$ for $\alpha I e_{v}$ and $K_{\alpha}=K$ for $\alpha K e_{v}$.

Now the left hand side of (2.11) is well defined, and we will start rewriting it as a sum of elements of $J_{S}$.

First of all, each term $\mu(\tau(\alpha)(\gamma))$ can be uniquely rewritten as $\mu\left(\tau\left(\gamma^{\prime}\right)\left(\alpha^{\prime}\right)\right)$ creating two new edges of $\tau(\alpha)(\gamma)$ in reverse order.

More formally, let $\tau\left(\gamma^{\prime}\right)$ be the result of contracting the edge $f=f_{\alpha}$ in $\tau(\alpha)(\gamma)$. It can be obtained from $\tau$ by inserting a new edge $g$ breaking the set of branches at the vertex $v$ into two parts. This edge has a common vertex with $e$. Denote by $w=w_{\gamma^{\prime}}$ another vertex of $g$. The tree $\tau\left(\gamma^{\prime}\right)\left(\alpha^{\prime}\right)$ is obtained from $\tau\left(\gamma^{\prime}\right)$ by inserting an edge breaking the branches at $w$ into two parts.

Second, we will show that the $\operatorname{sign} \epsilon(\alpha ; I, J, K, L)$ in $(2.11)$ can be rewritten as $\epsilon\left(\alpha^{\prime} ; I^{\prime}, J^{\prime}, K^{\prime}, L^{\prime}\right)$ where the flags $I^{\prime}, J^{\prime}, K^{\prime}, L^{\prime}$ at $w$ will depend on $\gamma^{\prime}$ but not on $\alpha^{\prime}$ and form an allowed quadruple.

Then (2.11) can be rewritten as

$$
\sum_{\gamma^{\prime}} \sum_{\alpha^{\prime}} \epsilon\left(\alpha^{\prime} ; I^{\prime}, J^{\prime}, K^{\prime}, L^{\prime}\right) \mu\left(\tau\left(\gamma^{\prime}\right)\left(\alpha^{\prime}\right)\right)
$$

It will remain only to convince ourselves that $\alpha^{\prime}$ runs over all 2-partitions of $F_{\tau\left(\gamma^{\prime}\right)}\left(w_{\gamma^{\prime}}\right)$.

We will dogmatically describe the choices involved. We checked that everything fits together by drawing twelve diagrams exhausting all possible mutual positions of $I, J, K, L, e, f, g$ in the trees $\tau(\alpha)(\gamma)=\tau\left(\gamma^{\prime}\right)\left(\alpha^{\prime}\right)$.

The diagrams show that $\gamma^{\prime}$ breaks $I, J, K, L$ in such a way that either all four flags get in the same part, or one of them is taken apart. In the first case we simply choose $I^{\prime}=I, J^{\prime}=j, K^{\prime}=K, L^{\prime}=L$. In the second case we replace the flag that is taken apart by the appropriate flag belonging to the edge $g$. One easily sees that the resulting quadruples are allowed.

\section{(v) $\sigma$ breaks $\tau$ at $v$}

Again, we will consider several cases depending on how $\sigma$ breaks $I, J, K, L$. There are four basic options: $\sigma I J K L, I \sigma J K L, J \sigma I K L, I J \sigma K L$. All other possibilities either can be obtained from one of these by exchanging $J$ and $L, I$ and $K$, or else refer to those $\sigma$ for which $l_{\sigma} r(\tau, v ; I, J, K, L)=0$. The latter happens when $I K \sigma J L$, because then $\sigma$ cannot break any $\tau(\alpha)$ with either $I J \alpha K L$ or $I J \alpha K L$. 
Assume first that one of the options $\sigma I J K L, I \sigma J K L, J \sigma I K L$ holds. Denote by $w$ the vertex of $\sigma * \tau$ incident to three or more of the flags $I, J, K, L$.

As in (2.12), we can rewrite the nonvanishing terms of $l_{\sigma} r(\tau, v ; I, J, K, L)$ as follows:

$$
\epsilon(\alpha ; I, J, K, L) l_{\sigma} \mu(\tau(\alpha))=\epsilon\left(\alpha^{\prime} ; I^{\prime}, J^{\prime}, K^{\prime}, L^{\prime}\right) \mu\left((\sigma * \tau)\left(\alpha^{\prime}\right)\right)
$$

Here $\alpha^{\prime}$ is a 2-partition of $F_{\sigma * \tau}(w)$, and $I^{\prime}, J^{\prime}, K^{\prime}, L^{\prime}$ are defined by the following prescription. If $I, J, K, L$ are all contained in the same part of $\alpha, I^{\prime}=I, J^{\prime}=$ $J, K^{\prime}=K, L^{\prime}=L$. If one of the flags is taken apart, it must be replaced by the flag of $e$ incident to $w$.

A straightforward check then shows that the summation over all $\alpha$ produces the same result as the summation over all $\alpha^{\prime}$, so that finally

$$
l_{\sigma} r(\tau, v ; I, J, K, L)=r\left(\sigma * \tau, w ; I^{\prime}, J^{\prime}, K^{\prime}, L^{\prime}\right) .
$$

It remains to consider the case $I J \sigma K L$. In this case, among the summands of $r(\tau, v ; I, J, K, L)$ exactly one has the property $\tau\left(\alpha_{0}\right)=\sigma * \tau$. To multiply it by $l_{\sigma}$, we use the formula (2.5), in which we have to replace $\tau$ by $\tau\left(\alpha_{0}\right)$ and rename the summation variables, say, to $\beta, \gamma$. However, we will use our current $I, J, K, L$ in the same sense as in (2.5), so that finally we get

$$
l_{\sigma} \mu\left(\tau\left(\alpha_{0}\right)\right)=-\sum_{\beta: I J \beta e_{1}} \mu((\sigma * \tau)(\beta))-\sum_{\gamma: e_{2} \gamma K L} \mu((\sigma * \tau)(\gamma))
$$

On the other hand, the nonvanishing terms $l_{\sigma} \mu(\tau(\alpha))$ with $\alpha \neq \alpha_{0}$ can be represented as $\mu((\sigma * \tau)(\delta))$ where $\delta$ breaks $\sigma * \tau$ at one of the vertices of $e$. A contemplation shows that these terms exactly cancel $(2.15)$.

2.3.4. Multiplications by $l_{\sigma}$ pairwise commute $\bmod J_{S}$. We want to prove that

$$
l_{\sigma_{1}}\left(l_{\sigma_{2}} \mu(\tau)\right) \equiv l_{\sigma_{2}}\left(l_{\sigma_{1}} \mu(\tau)\right) \bmod J_{S} .
$$

There is a long list of subcases that have to be treated separately: $\sigma_{1}$, resp. $\sigma_{2}$, can break $\tau$ at a vertex $v_{1}$, resp. $v_{2}$, an edge $e_{1}$, resp. $e_{2}$, or not to break $\tau$. For each of the possible breaking combinations, if the surgery loci are not incident, say, $v_{1}$ is not a vertex of $e_{2}$, the reasoning is straightforward, but it becomes more tedious otherwise.

In the section A.3 of the Appendix to [LoMa], we displayed the relevant calculations for the case when the painted set $S$ has exactly two white labels. In this case edges of $\tau$ have to form a linear chain, with two white labels attached at the respective end vertices. 
The total number of white labels influences everything in our definitions: the total supply of painted stable trees, the formulas for multiplication, and the list of the generators of $I_{S}$ and $J_{S}$. Therefore generally we cannot simply refer to the Appendix in [LoMa]. However, a check of pairwise commutativity of $l_{\sigma}$ 's does allow such a reduction.

Let us illustrate this in the case numbered $(i i)\left(i i^{\prime}\right)$ in A.3, [LoMa]: $\sigma_{1}$, resp. $\sigma_{2}$, breaks $\tau$ at the edges $e_{1}$, resp. $e_{2}$, having a common vertex. Let us replace $\tau$ by the linear tree $\tau_{0}$ with edges $e_{1}, e_{2}$. Besides halves of these edges, we endow $\tau_{0}$ with tails which are branches of $\tau$ incident to the respective three vertices. At the two end vertices of $\tau_{0}$, choose two branches of $\tau$ which carry white labels and declare the respective tails of $\tau_{0}$ white. Declare other tails black. Replace the 2-partitions $\sigma_{1}, \sigma_{2}$, by the induced partitions of tails of $\tau_{0}$.

Now, the calculations in A.3 referring to $\tau_{0}$ are compatible with the respective calculations for $\tau$, because the formula (2.5) is "local". They allow less freedom with respect to the choice of $I, J, K, L$, but this is inessential, because from 2.3.2 and 2.3.3 it follows that any choice will do.

Finally, with the choices made in [LoMa], we get (2.16) as an exact equality.

We have now proved that $\mathcal{R}_{S}$ acts upon $H_{* S}$. It remains to check that $I_{S}$ annihilates $H_{* S}$.

2.3.5. Elements $R_{i j k l}$ annihilate $H_{* S}$. The case when all labels are white was treated in [Ma1], Chapter III, 4.8.4. With very minor additional precautions, the treatment can be repeated for general painted $S$.

The reason is that, although we have a short supply of painted stable 2-partitions $\sigma$ 's if not all labels are white, nevertheless for an allowed quadruple $i, j, k, l \subset S$, the relation $R_{i j k l}$ involves with non-zero coefficients exactly those $\sigma$ 's which are simply stable. Painted stable trees also are simply stable. Finally, the multiplication formulas (2.5) written for allowed quadruples $I, J, K, L$ constitute a part of the multiplication formulas in the unpainted case.

In [Ma1], III.4.8.4, we show that $R_{i j k l}[\mu(\tau)]=0$, again for different reasons in different combinatorial situations. When no choices are involved in a calculation, it is valid in the painted case as well. If a choice of $I, J, K, L$ is involved, one can check that for an allowed $i, j, k, l$, the choices made in [Ma1] are allowed as well.

2.3.6. Elements $R_{\sigma_{1} \sigma_{2}}$ annihilate $H_{* S}$. These elements are $l_{\sigma_{1}} l_{\sigma_{2}}$ such that $\sigma_{1}$ and $\sigma_{2}$ do not break each other. If one of them, say, $\sigma_{1}$, does not break $\tau$ either, then because of commutativity $l_{\sigma_{1}} l_{\sigma_{2}} \mu(\tau)=0$.

If both of them break $\tau$, they have to break $\tau$ at one and the same vertex $v$. It is then easy to check that $l_{\sigma_{1}} l_{\sigma_{2}} \mu(\tau)=0$. 


\section{$\S 3$. Cohomology and homology as functors on trees}

3.1. Groups $H^{*}(\tau)$ and $H_{*}(\tau)$. Let $\tau$ be a stably painted tree. We extend the painting of tails of $\tau$ to the painting of all flags declaring all halves of the edges white. Finally, we put

$$
H^{*}(\tau):=\otimes_{v \in V_{\tau}} H_{F_{\tau}(v)}^{*}, \quad H_{*}(\tau):=\otimes_{v \in V_{\tau}} H_{* F_{\tau}(v)} .
$$

For one-vertex trees, we recover the rings/modules (1.3), (2.2) with which we worked in the earlier sections. The tensor products (over $k$ ) of families of objects labeled by finite sets, as in (3.1), are defined generally in symmetric monoidal categories and allow one to make explicit the functorial properties with respect to the maps of the index sets. In particular, $H^{*}(\tau)$, resp. $H_{*}(\tau)$, has a natural structure of $k$-algebra, resp. $H^{*}(\tau)$-module, with compositions defined componentwise.

The main result of this section is:

3.2. Theorem. For any contraction morphism $f: \tau^{\prime} \rightarrow \tau$ of stably painted trees, one can define a ring homomorphism

$$
f^{*}: H^{*}(\tau) \rightarrow H^{*}\left(\tau^{\prime}\right)
$$

and a compatible homomorphism of modules

$$
f_{*}: H_{*}\left(\tau^{\prime}\right) \rightarrow H_{*}(\tau)
$$

which makes $H^{*}$ and $H_{*}$ functors on the category of trees and contractions.

Functors $H^{*}, H_{*}$ are well determined by their actions on isomorphisms (obvious) and on the morphisms contracting a unique edge of an one-edge tree. These restrictions are explicitly described below.

Proof. Let $S$ be a painted stable set identified with an one-vertex tree with flags $S, \sigma$ an one-edge painted stable $S$-tree identified with a 2 -partition $S=S_{1} \cup S_{2}$. We denote by $e$ the edge of $\sigma$, and by $v_{1}, v_{2}$ its vertices carrying flags $S_{1}, S_{2}$, and by $e_{1}, e_{2}$ the respective halves of $e$. Denote by $f_{e}$ the contraction morphism. We want first of all to define a ring homomorphism $f_{e}^{*}: H_{S}^{*} \rightarrow H_{S_{1} \cup\left\{e_{1}\right\}}^{*} \otimes H_{S_{2} \cup\left\{e_{2}\right\}}^{*}$

We start with describing its (depending on some auxiliary choices) lift $\phi_{e}^{*}: \mathcal{R}_{S} \rightarrow$ $\mathcal{R}_{S_{1} \cup\left\{e_{1}\right\}} \otimes \mathcal{R}_{S_{2} \cup\left\{e_{2}\right\}}$. Put $\phi_{e}^{*}\left(l_{\rho}\right)=0$ if $\rho$ does not break $\sigma$. If $\rho$ breaks $\sigma$ at $v_{1}$, it defines a painted stable 2-partition $\rho_{1}$ of $S_{1}$ and the respective new edge $f$ of $\rho * \sigma$. Let $S_{1}^{\prime}$ denote the set of flags of this tree at the common vertex of $e$ and $f$, and $S_{1}^{\prime \prime}$ the flags at the other vertex of $f$. Denote by $\rho_{1}$ the one edge $\left(S_{1} \cup\left\{e_{1}\right\}\right)$-tree with

tails $S_{1}^{\prime} \cup\left\{e_{1}\right\}, S_{1}^{\prime \prime}$. Similarly, if $\rho$ breaks $\sigma$ at $v_{2}$ define an one-edge $\left(S_{2} \cup\left\{e_{2}\right\}\right)$-tree $\rho_{2}$. Put

$$
\phi_{e}^{*}\left(l_{\rho}\right):=l_{\rho_{1}} \otimes 1, \text { resp. } 1 \otimes l_{\rho_{2}} \in \mathcal{R}_{S_{1 \cup\left\{e_{1}\right\}}} \otimes \mathcal{R}_{S_{2 \cup\left\{e_{2}\right\}}}
$$


It remains to define $\phi_{e}^{*}$ in the case when $\rho=\sigma$, or equivalently, when $\rho$ breaks $\sigma$ at $e$. In this case we choose an allowed quadruple of flags $i, j, k, l$ such that $i, j \in S_{1}, k, l \in S_{2}$, and define, similarly to (1.7), (2.5),

$$
\phi_{e}^{*}\left(l_{\sigma}\right)=-\sum_{\alpha: i j \alpha e_{1}} l_{\alpha} \otimes 1-\sum_{\beta: i j \beta e_{1}} 1 \otimes l_{\beta} \in \mathcal{R}_{S_{1 \cup\left\{e_{1}\right\}}} \otimes \mathcal{R}_{S_{2 \cup\left\{e_{2}\right\}}} .
$$

Notice right away that another choice of $i, j, k, l$ will not change the result modulo $I_{S_{1 \cup\left\{e_{1}\right\}}} \otimes \mathcal{R}_{S_{2 \cup\left\{e_{2}\right\}}}+\mathcal{R}_{S_{1 \cup\left\{e_{1}\right\}}} \otimes I_{S_{2 \cup\left\{e_{2}\right\}}}$. The same reasoning as in 2.3.2 shows this: we consider a replacement of one flag, say, $i^{\prime}$, and then the respective difference as in (2.6) can be identified with $r\left(\alpha, v_{1} ; i^{\prime}, j, i, e_{1}\right) \otimes 1$.

3.2.1. Lemma. (a) There is a well defined ring homomorphism

$$
f_{e}^{*}: H_{S}^{*} \rightarrow H_{S_{1} \cup\left\{e_{1}\right\}}^{*} \otimes H_{S_{2} \cup\left\{e_{2}\right\}}^{*}, \quad\left[l_{\rho}\right] \mapsto\left[\phi_{e}^{*}\left(l_{\rho}\right)\right]
$$

(ii) $f_{e}^{*}$ is surjective. More precisely, let $\tau_{1}$, resp. $\tau_{2}$, be a painted stable $\left(S_{1} \cup\left\{e_{1}\right\}\right)-$ tree, resp. $\left(S_{2} \cup\left\{e_{2}\right\}\right)$-tree. Denote by $\tau_{1} \bullet \tau_{2}$ the $S$-tree obtained by gluing $e_{1}$ to $e_{2}$. Then

$$
f_{e}^{*}\left(\left[m\left(\tau_{1} \bullet \tau_{2}\right)\right]\right)=\left[m\left(\tau_{1}\right)\right] \otimes\left[m\left(\tau_{2}\right)\right] .
$$

Proof of Lemma 3.2.1. We must show that $\phi_{e}^{*}$ maps the generators (1.1) and (1.2) of $I_{S}$ into $I_{S_{1} \cup\left\{e_{1}\right\}}^{*} \otimes \mathcal{R}_{S_{2} \cup\left\{e_{2}\right\}}^{*}+\mathcal{R}_{S_{1} \cup\left\{e_{1}\right\}}^{*} \otimes I_{S_{2} \cup\left\{e_{2}\right\}}^{*} \cdot$

Start with a generator $R_{i j k l}$ which we will now endow with a superscript $S$. Our reasoning depends on the mutual position of $i, j, k, l$ and $\sigma$.

Case $i j k l \sigma$. If, say, $i, j, k, l \in S_{2}$, we have

$$
\phi_{e}^{*}\left(R_{i j k l}^{S}\right)=1 \otimes R_{i j k l}^{S_{2} \cup\left\{e_{2}\right\}} .
$$

Case $j \sigma i k l$. If $i, k, l \in S_{2}$, we have

$$
\phi_{e}^{*}\left(R_{i j k l}^{S}\right)=1 \otimes R_{i e_{2} k l}^{S_{2} \cup\left\{e_{2}\right\}} .
$$

Case $i \sigma j k l$. If $j, k, l \in S_{2}$, we have

$$
\phi_{e}^{*}\left(R_{i j k l}^{S}\right)=1 \otimes R_{e_{2} j k l}^{S_{2} \cup\left\{e_{2}\right\}} .
$$

Case $i k \sigma j l$. In this case

$$
\phi_{e}^{*}\left(R_{i j k l}^{S}\right)=0 .
$$


Case $i j \sigma k l$. In this case one summand of $R_{i j k l}^{S}$ is $l_{\sigma}$. Applying $\phi_{e}^{*}$ to it with the same choice of $i, j, k, l$ we get the expression (3.5). Applying $\phi_{e}^{*}$ to all other terms of $R_{i j k l}^{S}$ and using (3.4), we get the same terms as in (3.5), with reverse signs. Hence finally

$$
\phi_{e}^{*}\left(R_{i j k l}^{S}\right)=0
$$

Up to obvious symmetries, we have exhausted all possible alternatives. It remains to show that

$$
\phi_{e}^{*}\left(l_{\sigma_{1}}\right) \phi_{e}^{*}\left(l_{\sigma_{2}}\right) \in I_{S_{1} \cup\left\{e_{1}\right\}} \otimes \mathcal{R}_{S_{2} \cup\left\{e_{2}\right\}}+\mathcal{R}_{S_{1} \cup\left\{e_{1}\right\}} \otimes I_{S_{2} \cup\left\{e_{2}\right\}}
$$

if $\delta\left(\sigma_{1}, \sigma_{2}\right)=2$. We leave this as an exercise to the reader.

We have thus established that $\phi_{e}^{*}$ induces a ring homomorphism $f_{e}^{*}$. Representing $m\left(\tau_{1} \bullet \tau_{2}\right)$ as the product of generators corresponding to the edges, and applying (3.4), we get (3.7). This shows the surjectivity of $f_{e}^{*}$ and completes the proof of the lemma.

We now continue the proof of the Theorem 3.2 and turn to $H_{*}$. Motivated by (3.7) (and, of course, by algebraic geometry), we define (in the notations of Lemma 3.2 .1 (ii)):

$$
f_{e *}: H_{* S_{1} \cup\left\{e_{1}\right\}} \otimes H_{* S_{2} \cup\left\{e_{2}\right\}} \rightarrow H_{* S}, \quad\left[\mu\left(\tau_{1}\right)\right] \otimes\left[\mu\left(\tau_{2}\right)\right] \mapsto\left[\mu\left(\tau_{1} \bullet \tau_{2}\right)\right] .
$$

Compatibility of this prescription with the defining relations of $J_{S}$ (cf. (2.1)) is straightforward.

Since $[\mu(\tau)]=[m(\tau)] \mathbf{1}$ (cf. the proof of 2.3.1), combining (3.7) and (3.8) we obtain

$$
f_{e *}\left(f_{e}^{*}\left(\left[m\left(\tau_{1} \bullet \tau_{2}\right)\right]\right)(\mathbf{1} \otimes \mathbf{1})\right)=\left[m\left(\tau_{1} \bullet \tau_{2}\right)\right] \mathbf{1} .
$$

This is the key special case of the compatibility of $f_{e}^{*}$ and $f_{e *}$ ("projection formula" of algebraic geometry). The general case (obtained by replacing $\mathbf{1} \otimes \mathbf{1}$ with any element of $H_{*} \otimes H_{*}$ ) follows from it formally, because $\mathbf{1}$ is a free generator of $H_{*}$ over the respective $H^{*}$.

We can now define (3.2) and (3.3) by decomposing $f$ into a product of edge contractions and a final isomorphism and using (3.7), (3.8). Edge contractions commute in an intuitively evident sense, so that checking that the result is independent of the decomposition involves only a careful bookkeeping. We leave this as an exercise to the reader.

\section{$\S 4$. $\mathcal{L}$-algebras}

In this section we will define several versions of the notion of "algebra over extended modular operad of genus zero". The basic definition we start with is 
modeled upon the functorial treatment introduced in [KoMa], sec. 6. It involves unoriented painted stable trees and (combinatorial) cohomology algebras, and it produces a version of cyclic algebras in the sense of Getzler and Kapranov. Tensor product of algebras appears in the most straightforward way in this construction.

Other versions involve oriented trees and/or homology algebras. The generic name we use for all these versions is $\mathcal{L}$-algebras.

Finally, we will explain how the classical operads appear in this context.

4.1. From trees to tensors: unoriented case. Let $\mathcal{A}=\{T ; F,()$,$\} be a$ triple consisting of two free (or projective) finite rank $\mathbf{Z}_{2}$-graded $k$-modules $T, F$ and a (super)symmetric scalar product $($,$) on F$. We assume that $($,$) induces an$ isomorphism $F \rightarrow F^{t}$ where $F^{t}$ is the graded dual to $F$. We denote by $\Delta \in F \otimes F$ the respective Casimir element.

Let $\tau$ be a painted stable tree. Denote by $W_{\tau}$, resp. $B_{\tau}$, the set of its white, resp. black flags (recall that halves of edges are all white.) If $v$ is a vertex of $\tau$, $W_{\tau}(v)$, resp. $B_{\tau}(v)$, denotes the set of white, resp. black, flags incident to this vertex. Put

$$
\mathcal{A}(\tau):=T^{\otimes B_{\tau}} \otimes F^{\otimes W_{\tau}}=\otimes_{v \in V_{\tau}} T^{\otimes B_{\tau}(v)} \otimes F^{\otimes W_{\tau}(v)} .
$$

Thus, we attach the "white space" $F$ at each white flag, the "black space" $T$ at each black flag, and take the tensor product of all spaces.

Let now $f: \tau \rightarrow \sigma$ be a contraction. It identifies $F_{\sigma}$ with a subset $F_{\tau}^{s}$ of noncontracted flags. The complement consist of the halves of contracted edges $E_{\tau}^{c}$. We can define a natural map

$$
f_{\mathcal{A}}^{*}: \mathcal{A}(\sigma) \rightarrow \mathcal{A}(\tau)
$$

which tautologically identifies spaces attached to the flags which are paired by $f$ and then tensor multiplies the result by $\Delta^{\otimes E_{\tau}^{c}} \in(F \otimes F)^{\otimes E_{\tau}^{c}}$.

4.1.1. Proposition. In this way $\tau \mapsto \mathcal{A}(\tau)$ becomes a contravariant functor on the category of stably painted trees and contractions.

This is straightforward.

4.2. Definition. The structure of a cyclic $\mathcal{L}$-algebra upon $\mathcal{A}=\{T ; F,()$,$\} is a$ morphism of functors compatible with gluing

$$
I: \mathcal{A} \rightarrow H^{*}
$$

In other words, it consists of a family of maps indexed by stably painted trees

$$
I(\tau): T^{\otimes B_{\tau}} \otimes F^{\otimes W_{\tau}} \rightarrow H^{*}(\tau)
$$


such that for any contraction morphism $f: \tau \rightarrow \sigma$ we have

$$
I(\tau) \circ f_{\mathcal{A}}^{*}=f^{*} \circ I(\sigma): T^{\otimes B_{\sigma}} \otimes F^{\otimes W_{\sigma}} \rightarrow H^{*}(\tau)
$$

and moreover

$$
I\left(\tau_{1} \bullet \tau_{2}\right)=I\left(\tau_{1}\right) \otimes I\left(\tau_{2}\right): \mathcal{A}\left(\tau_{1}\right) \otimes \mathcal{A}\left(\tau_{2}\right) \rightarrow H^{*}\left(\tau_{1}\right) \otimes H^{*}\left(\tau_{2}\right)=H^{*}\left(\tau_{1} \bullet \tau_{2}\right)
$$

Restricting this definition to the case of pure white painted trees, we get the structure on $F$ which was called an operadic tree level Cohomological Field Theory in [KoMa], Def. 6.10. This is the same as an algebra over the cyclic operad $\left\{H_{*}\left(\bar{M}_{0, n+1}, k\right)\right\}$. The case of trees with exactly two white flags (and orientation, cf. below) produces a structure which is essentially a representation of a certain algebra $H_{*} T$ on $F$ studied in [LoMa], sec. 3.3-3.6. Thus, the notion of an $\mathcal{L}$-algebra combines both these structures.

4.2.1. Tensor product of cyclic $\mathcal{L}$-algebras. Let $\mathcal{A}=\left\{T_{1} ; F_{1},(,)_{1}\right\}, I_{1}$, resp. $\mathcal{B}=\left\{T_{2} ; F_{2},(,)_{2}\right\}, I_{2}$, be two cyclic $\mathcal{L}$-algebras. Put

$$
\begin{gathered}
\mathcal{A} \otimes \mathcal{B}:=\left\{T_{1} \otimes T_{2} ; F_{1} \otimes F_{2},(,)_{1} \otimes(,)_{2}\right\} \\
I_{\mathcal{A} \otimes \mathcal{B}}(\tau):=M \circ\left(I_{1} \otimes I_{2}\right)(\tau):
\end{gathered}
$$

where $M: H^{*}(\tau) \otimes H^{*}(\tau) \rightarrow H^{*}(\tau)$ is the ring multiplication. Compatibility with (4.5), (4.6) is straightforward.

4.2.2. Economy class descriptions of cyclic algebras. Let us consider only maps (4.4) indexed by one-vertex trees, with a painted set of flags $S=W \cup B$ :

$$
I_{S}: T^{\otimes B} \otimes F^{\otimes W} \rightarrow H_{S}^{*}
$$

The axiom (4.7) puts certain restrictions upon this family of maps. Namely, for any painted stable partition $S=S_{1} \cup S_{2}, S_{i}=W_{i} \cup B_{i}$, the following diagram must be commutative:

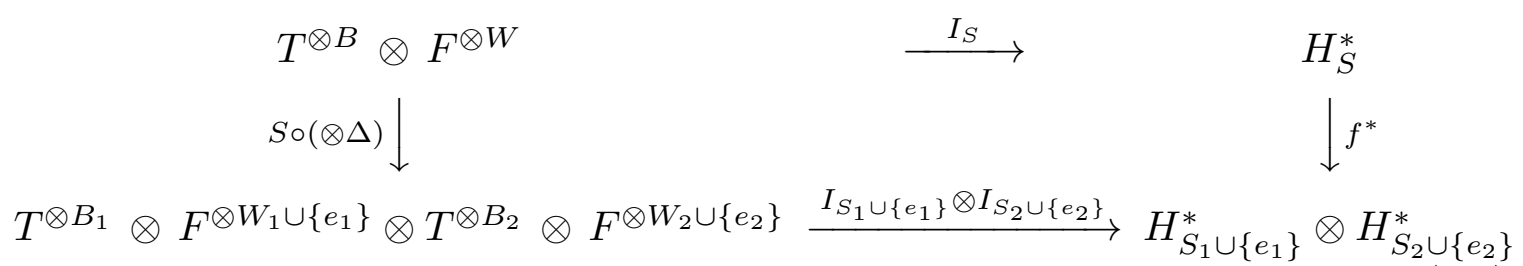

Here $f^{*}$ is the map defined by $(3.6),(3.7)$, and $S$ is an obvious reshuffling of factors. 
Moreover, any painted isomorphism $S \rightarrow S^{\prime}$ produces isomorphisms of the respective corners of the diagram (4.8), and we require the resulting cubic diagram to be commutative.

One easily sees that, conversely, given $I_{S}$ satisfying (4.8) and compatible with isomorphisms, we can uniquely reconstruct the structure of cyclic $\mathcal{L}$-algebra on $\mathcal{A}=\{T ; F,()$,$\} .$

We can further restrict ourselves to the subcategory of painted sets $S_{n, m}$ in which $W$ (resp. $B$ ) consists of an initial segment of the natural numbers $\{1, \ldots, n\}$ (resp. an initial segment of another copy of the natural numbers $\{\overline{1}, \ldots, \bar{m}\}$. Isomorphisms will reduce to the actions of two copies of symmetric groups $\mathbf{S}_{n} \times \mathbf{S}_{m}$. The axioms we get in this way are extensions of ones that are stated in $[\mathrm{KoMa}]$, Definition 6.1.

4.3. From trees to tensors: oriented case. An orientation of a painted stable root tree $\tau$ is uniquely determined by a choice of its root: an arbitrary white flag $r$. In the geometric realization, we then orient $r$ away from its vertex, and each other white flag in the direction of $r$. Put $W_{\tau}=W_{\tau}^{+} \cup W_{\tau}^{-}$where $W_{\tau}^{+}$consists of flags oriented towards their vertices ("in"), and $W_{\tau}^{-}$of flags oriented in reverse direction ("out".) Notice that all black flags are oriented "in".

An oriented contraction of oriented trees is the same as a usual contraction which sends root to root.

To produce tensors associated with oriented trees we start with a pair of modules $\mathcal{A}_{0}=\{T, F\}$ as in 4.1 , but do not assume a scalar product given. We then denote by $\Delta \in F \otimes F^{t}$ the image of the identity morphism.

For an oriented tree $\tau$ we put

$$
\mathcal{A}_{0}(\tau):=T^{\otimes B_{\tau}} \otimes\left(F^{t}\right)^{\otimes W_{\tau}^{-}} \otimes F^{\otimes W_{\tau}^{+}}
$$

Let now $f: \tau \rightarrow \sigma$ be an oriented contraction. As in 4.1, we can define a natural map

$$
f_{\mathcal{A}_{0}}^{*}: \mathcal{A}_{0}(\sigma) \rightarrow \mathcal{A}_{0}(\tau)
$$

which tautologically identifies spaces attached to the flags which are paired by $f$ and then tensor multiplies the result by $\Delta^{\otimes E_{\tau}^{c}} \in\left(F \otimes F^{t}\right)^{\otimes E_{\tau}^{c}}$.

4.3.1. Proposition. In this way $\tau \mapsto \mathcal{A}_{0}(\tau)$ becomes a contravariant functor on the category of oriented stably painted trees and contractions.

4.4. Definition. The structure of an $\mathcal{L}$-algebra upon $\mathcal{A}_{0}=\{T, F\}$ is a morphism of functors compatible with gluing

$$
I: \mathcal{A}_{0} \rightarrow H^{*} \text {. }
$$


4.5. $\mathcal{L}$-algebras in terms of combinatorial homology and correlators. We will now introduce one more version of $\mathcal{L}$-algebras involving homology and unoriented trees. It is this version which is most convenient for the passage to the differential equations.

For a change, we will start with an economy class description. We fix $\mathcal{A}_{0}=$ $\{T, F\}$ as in 4.3 .

4.5.1. Data. Consider one-vertex oriented stable painted trees. Such a tree is the same as a finite set of its flags represented in the form $S=(W \cup\{0\}) \cup B$, $|W \cup B| \geq 2$, where 0 is the root, $W \cup\{0\}$ (resp. $B$ ) is the subset of white (resp. black) flags. For each such $S$ we assume given an even homomorphism of $k$-modules (correlator)

$$
C_{S}: H_{* S} \otimes T^{\otimes B} \otimes F^{\otimes W} \rightarrow F
$$

This should be compared to (4.7): to pass from (4.7) to (4.11), we make a partial dualization: cohomology becomes homology and moves to the left hand side, whereas the factor $F^{t}$ corresponding to the root becomes $F$ and moves to the right hand side.

Once again, we can restrict ourselves by the sets $A=\{0,1, \ldots, n\}, B=\{\overline{1}, \ldots, \bar{m}\}$ taken from two disjoint copies of the set of nonnegative integers.

Since $H_{* S}$ is spanned by $[\mu(\tau)]$ where $\tau$ runs over unoriented stable painted $S$-trees, to give (4.11) is the same as to give a family of maps

$$
C_{S}([\mu(\tau)]): T^{\otimes B} \otimes F^{\otimes W} \rightarrow F
$$

Notice again that the orientation is encoded by $S$ which is given together with the choice of (a label of the) root.

The intuitive meaning of (4.12) is this: given a rooted tree $\tau$, we consider it as the flowchart of a computation. Concretely, an input assigns an element $f_{a}$ of $F$ (resp. $t_{b}$ of $T$ ) to each white non-root tail $a$ (resp. to each black tail $b$ ). Then the output is an element

$$
C_{S}([\mu(\tau)])\left(\otimes_{b} t_{b} \otimes_{a} f_{a}\right) \in F .
$$

We associate this output with the root of $\tau$.

4.5.2. Axioms. (A) Operadic relations. If we graft two (or more) oriented trees by gluing root(s) to white tail(s), the resulting tree must be the flowchart for the composition of the respective functions.

Notice that since black tails are never glued, elements of $T$ may be considered as "parameters", whereas the actual arguments (and values) of the correlators belong to $F$. 
(B) Symmetry relations. They say that the product $\mathbf{S}_{W} \times \mathbf{S}_{B}$ of the permutation groups of $W$ and $B$ acting upon (4.11) in an evident way leaves these maps invariant. Notice that the action on $H_{* S}$ is also involved in this requirement.

(C) Linear relations. They assure us that all linear relations between $[\mu(\tau)]$ in $H_{* S}$ imply the respective relations between correlators. We can restrict ourselves by the basic relations (2.1), or their cohomological version (1.10). Namely, starting with an $S$-tree $\tau$, its vertex $v$, and an allowed quadruple of flags $I, J, K, L$ at this vertex, as in 1.4, we must have

$$
\sum_{\alpha} \epsilon(\alpha ; I, J, K, L) C_{S}([\mu(\tau(\alpha))])=0
$$

where we sum over all 2 -partitions of the set of flags at $v$, and $\varepsilon=1,-1$, or 0 , depending on whether $I J \alpha K L, K J \alpha I L$ or none of it.

4.6. Top correlators. Let $\tau$ be an one vertex tree whose tails are labeled by $(W \cup\{0\}) \cup B$. For $W=\{1, \ldots, n+1\}, B=\{\overline{1}, \ldots, \bar{m}\}$, we will write $c\left(t_{1}, \ldots, t_{m}, f_{1}, \ldots, f_{n+1}\right)$ in place of $(4.13)$ and call these elements the top correlators. They are symmetric in $T$ - and $F$-arguments separately. We will formally extend them to polylinear functions symmetric in all variables. All the operadic relations follow by iteration from this symmetry and from the following ones:

$$
\begin{gathered}
c\left(t_{1}, \ldots, t_{m}, f_{1}, f_{2}, \ldots, f_{n}, c\left(t^{\prime}{ }_{1}, \ldots, t_{q}^{\prime} ; f^{\prime}{ }_{1}, \ldots, f^{\prime}{ }_{p+1}\right)\right)= \\
c\left(t_{1}, \ldots, t_{m}, f_{1}, f_{2}, \ldots, f_{n}, t^{\prime}{ }_{1}, \ldots, t^{\prime}{ }_{q}, f^{\prime}{ }_{1}, \ldots, f_{p+1}^{\prime}\right)
\end{gathered}
$$

Now fixing all the arguments except for $f_{n+1}$, consider $c\left(t_{1}, \ldots, t_{m}, f_{1}, \ldots, f_{n+1}\right)$ as a function of $f_{n+1}$, that is, an element of End $F$ (even or odd, depending on the sum of parities of the fixed arguments), and denote it by

$$
\left\langle t_{1}, \ldots, t_{m}, f_{1}, \ldots, f_{n}\right\rangle \in \text { End } F \text {. }
$$

Again, we will allow arbitrary permutations of arguments in this expression, followed by the standard sign change, and call the resulting expressions top matrix correlators. Then the grafting relations (4.15) turn into the simple factorization relations in End $F$ :

$$
\begin{gathered}
\left\langle t_{1}, \ldots, t_{m}, f_{1}, f_{2}, \ldots, f_{n}, t^{\prime}{ }_{1}, \ldots, t^{\prime}{ }_{q}, f^{\prime}{ }_{1}, \ldots, f^{\prime}{ }_{p}\right\rangle= \\
\left\langle t_{1}, \ldots, t_{m}, f_{1}, f_{2}, \ldots, f_{n}\right\rangle\left\langle t^{\prime}{ }_{1}, \ldots, t^{\prime}{ }_{q}, f^{\prime}{ }_{1}, \ldots, f^{\prime}{ }_{p}\right\rangle .
\end{gathered}
$$

We can now formulate the first result describing $\mathcal{L}$-algebras in terms of their top matrix correlators. In order to make connection with results of [LoMa], we introduce the following notation. Choose bases of $F, T$ and let their union, which is a basis of $T \oplus F$, be $\left\{\Delta_{i}\right\}$, for some index set $I$. Fix a structure of the (oriented, homological) $\mathcal{L}$-algebra upon $(T, F)$ and consider its top matrix correlators for the elements of the chosen basis. 
4.6.1. Theorem. (i) Top matrix correlators

$$
\left\langle\Delta_{a_{1}} \ldots \Delta_{a_{n}}\right\rangle \in \operatorname{End} F, \quad n \geq 2
$$

are (super)symmetric in their arguments. Furthermore, for any allowed quadruple $i, j, k, l \in I$

$$
\sum_{\sigma: i j \sigma k l} \varepsilon\left(\sigma,\left(a_{m}\right)\right)\left\langle\prod_{m \in \sigma_{1}} \Delta_{a_{m}}\right\rangle \cdot\left\langle\prod_{m \in \sigma_{2}} \Delta_{a_{m}}\right\rangle-\sum_{\sigma: k j \sigma i l} \varepsilon\left(\sigma,\left(a_{m}\right)\right)\left\langle\prod_{m \in \sigma_{1}} \Delta_{a_{m}}\right\rangle \cdot\left\langle\prod_{m \in \sigma_{2}} \Delta_{a_{m}}\right\rangle=0 .
$$

Here $\sigma$ runs over ordered 2-partitions of $\{1, \ldots, n\}$. We choose additionally an arbitrary ordering of both parts $\sigma_{1}, \sigma_{2}$ determining the ordering of $\Delta$ 's in the angular brackets, and compensate this choice by the \pm 1 -factor $\varepsilon\left(\sigma,\left(a_{k}\right)\right)$.

(ii) Conversely, any family of elements $\left\langle\Delta_{a_{1}} \ldots \Delta_{a_{n}}\right\rangle \in$ End $F$ defined for all $n \geq 2$ and $\left(a_{1}, \ldots, a_{n}\right) \in I^{n}$ and satisfying the symmetry conditions and (4.17), is the family of top matrix correlators of a unique structure of $\mathcal{L}$-algebra on $(T, F)$.

Proof. (i) Both symmetry properties follow from the definitions, whereas (4.17) follows from (4.14), written for one-vertex trees $\tau$ and transformed with the help of (4.16).

(ii) If we know all top matrix correlators, we can recursively determine maps (4.13) for all stable trees $\tau$ with $e \geq 1$ edges. In fact, choose an end edge of $\tau$ and cut it, thus representing $\tau$ as the result of grafting an one-vertex tree $\rho$ to a tree $\sigma$ with $e-1$ edges. Choose the flag $a$ of $\sigma$ that gets grafted as special input and, using partial dualization, rewrite (4.13) as a map $T^{\otimes B} \otimes F^{\otimes W} \rightarrow$ End $F$, that is, a matrix correlator related to the tree $\sigma$. From the grafting axiom (A) above it follows that (4.13) is uniquely calculated from the latter matrix correlator and the top matrix correlator for $\rho$, via a generalization of the factorization relations (4.16).

This shows that the $\mathcal{L}$-structure on $(T, F)$ with given top matrix correlators is unique, if it exists at all. In order to prove existence, we must check that all linear relations (4.14) follow from their special cases (4.17). The argument is essentially the same as in the proof on p. 462 of [LoMa]; the difference is that in [LoMa] the relevant products of top correlators were linear ordered, whereas here they are controlled by a tree, so a more careful bookkeeping is required. We leave this as an exercise.

\section{$\S 5$. Differential geometry and $\mathcal{L}$-algebras}

5.1. Notation. Consider a structure of $\mathcal{L}$-algebra upon $(T, F)$ determined by its top matrix correlators $\left\langle\Delta_{a_{1}} \ldots \Delta_{a_{n}}\right\rangle$ as in Theorem 4.6.1 (i). Denote by $\left(x^{a}\right)$ 
the linear coordinate system on $T \oplus F$ dual to $\left(\Delta_{a}\right)$. Put

$$
\mathcal{B}=\sum_{n=1}^{\infty} \sum_{\left(a_{1}, \ldots, a_{n}\right)} \frac{x^{a_{n}} \ldots x^{a_{1}}}{n !}\left\langle\Delta_{a_{1}} \ldots \Delta_{a_{n}}\right\rangle \in k[[x]] \otimes \text { End } F
$$

5.1.1. Theorem. a) We have

$$
d \mathcal{B} \wedge d \mathcal{B}=0
$$

b) Conversely, let $\mathcal{B} \in k[[x]] \otimes$ End $F$ be any even formal series without constant term satisfying the equation (5.2). Define its symmetrized coefficients $\Delta\left(a_{1}, \ldots, a_{n}\right)$, $a_{i} \in I$, by the following conditions: first,

$$
\mathcal{B}=\sum_{n=1}^{\infty} \sum_{\left(a_{1}, \ldots, a_{n}\right)} \frac{x^{a_{n}} \ldots x^{a_{1}}}{n !} \Delta\left(a_{1}, \ldots, a_{n}\right)
$$

second, the parity of $\Delta\left(a_{1}, \ldots, a_{n}\right)$ coincides with that of $x^{a_{n}} \ldots x^{a_{1}}$, and third,

$$
\Delta\left(a_{s(1)}, \ldots, a_{s(n)}\right)=\varepsilon\left(s,\left(a_{i}\right)\right) \Delta\left(a_{1}, \ldots, a_{n}\right)
$$

for any permutation $s$ of $\{1, \ldots, n\}$.

Then there exists a unique structure of $\mathcal{L}$-algebra upon $(T, F)$ with top correlators

$$
\left\langle\Delta_{a_{1}} \ldots \Delta_{a_{n}}\right\rangle=\Delta\left(a_{1}, \ldots, a_{n}\right)
$$

Theorem 4.6.1 shows that this is a particular case of the Proposition 3.6.1 of [LoMa]. Moreover, the discussion in 3.2 of [LoMa] establishes a bijection between the even formal solutions to the equation (5.2) and pencils of formal flat connections $\nabla_{0}+\lambda \mathcal{A}$ on the trivial vector bundle with fiber $F$ on the formal completion of $T \oplus F$ at zero.

In the remaining part of this section we will establish certain properties of solutions to (5.2) and related structures. As in 3.1-3.2 of [LoMa], we will work in a more general setting, allowing not necessarily formal manifolds as our base spaces.

5.2. Commutativity equations. Generally, let $M$ be a (super)manifold in one of the standard categories $\left(C^{\infty}\right.$, analytic, formal ...). Denote by $F$ a local system of finite-dimensional vector (super)spaces on $M$. This is essentially the same as a locally free sheaf $\mathcal{S}$ endowed with a flat connection $\nabla_{0}: \mathcal{S} \rightarrow \Omega_{M}^{1} \otimes_{\mathcal{O}_{M}} \mathcal{S}$ : from 
$\left(\mathcal{S}, \nabla_{0}\right)$ one gets $F:=\operatorname{Ker} \nabla_{0}$, and from $F$ one gets $\mathcal{S}:=\mathcal{O}_{M} \otimes F$ (tensor product over constants), $\nabla_{0}(t \otimes s)=d t \otimes_{\mathcal{O}_{M}} s$ for local sections $t \in \mathcal{O}_{M}, s \in F$.

Here $d$ is the de Rham differential, which extends in the standard way to $\Omega_{M}^{*}$, whereas $\nabla_{0}$ extends to the whole tensor algebra of $S$.

In particular, we have the induced flat connection denoted by the same letter $\nabla_{0}$ upon End $\mathcal{S}=\mathcal{O}_{M} \otimes$ End $F$.

An even section $B$ of $\mathcal{O}_{M} \otimes$ End $F$ is called a solution to the commutativity equations for $(M, F)$ if

$$
\nabla_{0} B \wedge \nabla_{0} B=0 .
$$

Our sign conventions are determined by postulating that $d$ and $\nabla_{0}$ are odd. We traditionally denote by $\wedge$ the multiplication in $\Omega_{M}^{*}$ and in the tensor products of $\Omega_{M}^{*}$ with other sheaves of algebras, although in the supergeometry this is slightly misleading: multiplication in $\Omega_{M}^{*}$ is supercommutative, not superalternate.

If we choose local coordinates $\left(t^{i}\right)$ in $M$ and a basis of flat (belonging to $F$ ) local sections of $\mathcal{S}, B$ becomes a matrix function $t \mapsto B(t)$, and $\nabla_{0} B$ becomes a matrix of 1 -forms $d B$ on $M:\left(\nabla_{0} B\right)_{i}^{j}=d B_{i}^{j}$ so that (5.2) is a particular case of (5.3).

Putting $d B=\sum_{i} d t^{i} b_{i}(t)$ where $t=\left(t^{i}\right)$, so that

$$
b_{i}(t):=\frac{\partial B}{\partial t^{i}} \in \mathcal{O}_{M} \otimes \text { End } F
$$

we can easily check that (5.3) can be written in the form of commutativity equations

$$
\forall i, j,\left[b_{i}(t), b_{j}(t)\right]=0
$$

where the brackets denote the supercommutator. Thus $b_{i}$ span over $\mathcal{O}_{M}$ a sheaf of (super)abelian Lie subalgebras of $\mathcal{O}_{M} \otimes$ End $F$. This sheaf is intrinsically associated with $B$; we will denote it $D B$.

5.2.1. Definition. (i) A solution $B$ to the commutativity equations as above is called maximal one, if $D B$ is a maximal subsheaf of (super)abelian Lie subalgebras of $\mathcal{O}_{M} \otimes$ End $F$ in the following sense: any local section $c(t) \in \mathcal{O}_{M} \otimes$ End $F$ (super)commuting with $D B$ belongs to $D B$.

(ii) A maximal solution is called strictly maximal if $D B$ is locally freely generated by $b_{i}(t)$.

Notice that if $T$ is the formal completions of a linear space at zero, maximality of $D B$ is equivalent to the maximality at zero. 
5.2.2. Pullback. Any morphism $\varphi: M^{\prime} \rightarrow M$ and any solution $B$ to the commutativity equations for $(M, F)$ produces the pullback solution $\varphi^{*}(B)$ for $\left(M^{\prime}, \varphi^{*}(F)\right)$.. Of course, if $M$ and $M^{\prime}$ are formal completions of linear spaces at zero, $\varphi$ is generally given by formal series with vanishing constant terms. If $\varphi$ is a closed embedding, we call $\varphi^{*}(B)$ the restriction of $B$, and conversely, we call $B$ a continuation of $\varphi^{*}(B)$ (both with respect to $\varphi$ ).

In particular, the automorphism group of $(M, F)$ acts upon the space of solutions. In the formal case, this is the group of formal invertible coordinate changes. Hence the linear structure of $M$ plays no role, and we can simply speak about formal manifolds.

5.2.3. Proposition. Let $B$ be a solution to the commutativity equations over a manifold $M$, and $B^{\prime}$ its continuation to $M^{\prime}$ with respect to a closed embedding $\iota: M \rightarrow M^{\prime}$. Denote by $\widehat{M}^{\prime}$ the formal completion of $M^{\prime}$ along $M$, and by $\widehat{B}^{\prime}$ the solution induced by $B^{\prime}$ on it.

(i) If $B$ is maximal, everywhere locally over $M$ there exists a formal projection $\varphi: \widehat{M}^{\prime} \rightarrow M, \varphi \circ \iota=i d_{M}$, such that $\widehat{B}^{\prime}=\varphi^{*}(B)$.

(ii) If $B$ is strictly maximal, these local formal projections are unique and hence glue together to a global formal projection $\hat{M}^{\prime} \rightarrow M$ with the same property.

Proof. Working in a local chart, choose local coordinates

$$
(t, \theta)=\left(t^{1}, \ldots, t^{m} ; \theta^{1}, \ldots, \theta^{n}\right)
$$

on $M^{\prime}$ such that $M$ in $M^{\prime}$ is given by the equations $\theta=0$. Choosing also a basis of sections of $F$, consider $B$ and $B^{\prime}$ as matrix functions. Denote by $\theta^{(k)}$ (super)symmetric monomials in $\theta$ where $(k)$ are polydegrees. We have

$$
d B \wedge d B=0, d B^{\prime} \wedge d B^{\prime}=0, B^{\prime}(t, 0)=B(t)
$$

Let $|k|$ be the total degree (sum of coordinates) of the polydegree $(k)$. To prove the statement (i), we have to find a family of local functions $\left(\lambda_{(k)}^{i}(t)\right), i=1, \ldots, m$, $|k| \geq 1$, on $M$, such that putting

$$
B_{N}^{\prime}(t, \theta):=B\left(t+\sum_{|k| \leq N} \lambda_{(k)}(t) \theta^{(k)}\right),
$$

we have

$$
B_{N}^{\prime}(t, \theta) \equiv B^{\prime}(t, \theta) \bmod \theta^{\geq N+1},
$$

where $\theta^{\geq N+1}$ is the ideal generated by $\theta^{(k)}$ with $|k| \geq N+1$. In fact, if we find such a family, the local formal projection $\varphi$ we look for is given by $\varphi^{*}\left(t^{i}\right)=$ 
$t^{i}+\sum_{(k)} \lambda_{(k)}^{i}(t) \theta^{(k)}$. Uniqueness of this family means uniqueness of the formal projection.

Obviously, $B_{0}^{\prime}(t, \theta)=B(t)$ is the only possible choice. In order to find $B_{1}^{\prime}(t, \theta)=$ $B\left(t+\sum_{j=1}^{n} \lambda_{j}(t) \theta^{j}\right)$ satisfying (5.7) notice that

$$
B\left(t+\sum_{j=1}^{n} \lambda_{j}(t) \theta^{j}\right) \equiv B(t)+\sum_{i=1}^{m} \sum_{j=1}^{n} \lambda_{j}^{i}(t) \theta^{j} b_{i}(t) \bmod \theta^{\geq 2}
$$

whereas

$$
B^{\prime}(t, \theta) \equiv B(t)+\left.\sum_{j=1}^{n} \frac{\partial B^{\prime}}{\partial \theta^{j}}\right|_{\theta=0} \theta^{j} \bmod \theta^{\geq 2}
$$

Hence $\lambda_{j}^{i}(t)$ exist iff all $\left.\frac{\partial B^{\prime}}{\partial \theta^{j}}\right|_{\theta=0}$ belong to $D B$ that is, (super)commute with $b_{i}(t)$. But this follows from $d B^{\prime} \wedge d B^{\prime}=0$ evaluated at $\frac{\partial}{\partial t^{i}} \wedge \frac{\partial}{\partial \theta^{j}}$. Moreover, $\lambda_{j}^{i}(t)$ are unique, if $D B$ is freely generated by $\left(b_{i}(t)\right)$.

This reasoning can be generalized to provide an inductive step from $N$ to $N+1$.

Namely, assume that we already found $B_{N}^{\prime}(t, \theta)$ of the form (5.6) satisfying $(5.7)$, $N \geq 1$. We need a vector $X_{N+1}=\left(X_{N+1}^{j}\right), j=1, \ldots, n$, whose coordinates are forms of degree $N+1$ in $\theta$ with coefficients depending on $t$ such that

$$
\begin{gathered}
B_{N+1}^{\prime}(t, \theta):=B\left(t+\sum_{|k| \leq N} \lambda_{(k)}(t) \theta^{(k)}+X_{N+1}\right) \equiv \\
\equiv B_{N}^{\prime}(t, \theta)+\sum_{j} X_{N+1}^{j} b_{j}\left(t+\sum_{|k| \leq N} \lambda_{(k)}(t) \theta^{(k)}\right) \bmod \theta^{\geq N+2} \\
\equiv B_{N}^{\prime}(t, \theta)+\sum_{j} X_{N+1}^{j} b_{j}(t) \bmod \theta^{\geq N+2}
\end{gathered}
$$

satisfies (5.7) with $N$ replaced by $N+1$. Let

$$
B^{\prime}(t, \theta) \equiv B_{N}^{\prime}(t, \theta)+Y_{N+1} \bmod \theta^{\geq N+2}
$$

where $Y_{N+1}$ is a form of degree $N+1$ in $\theta$ with coefficients depending on $t$. In order to establish the existence of $X_{N+1}$ it suffices to check as above that the coefficients of $Y_{N+1}$ commute with $b_{j}(t)$. In fact, since $d B^{\prime} \wedge d B^{\prime}=0$, we have

$$
d\left(B_{N}^{\prime}+Y_{N+1}\right) \wedge d\left(B_{N}^{\prime}+Y_{N+1}\right) \equiv 0 \bmod \theta^{\geq N+1}
$$


and since moreover $d B_{N}^{\prime} \wedge d B_{N}^{\prime}=0$ and $N \geq 1$, it follows that

$$
d Y_{N+1} \wedge d B_{N}^{\prime}+d B_{N}^{\prime} \wedge d Y_{N+1} \equiv 0 \bmod \theta^{\geq N+1} .
$$

Hence the coefficients of all derivatives $\frac{\partial}{\partial \theta^{j}} Y_{N+1}$ belong to $D B$. From Euler's formula it follows that the same holds for the coefficients of $Y_{N+1}$. Uniqueness in the case of strict maximality follows for this inductive step as well.

5.2.4. Primitive vectors. We keep the notation described above. Consider fibers of $F$ as (super)manifolds endowed with a linear structure. We can cover $M$ by open submanifolds such that over any chart $U$ we have a canonical trivialization $\left.F\right|_{U}=F_{0} \times U$, where $F_{0}$ is a fiber, or else the space of sections of $F$ over U.

An even section $h \in \Gamma(M, F)$ is called primitive (for the solution $B$ ) if the map of (super)manifolds $U \rightarrow F_{0}: t \mapsto B(t) h$ is a local isomorphism everywhere on $M$. An evident necessary condition for the existence of a primitive vector is the coincidence of (super)dimensions of $M$ and fibers of $F$.

5.3. Oriented associativity equations. Consider now a (super)manifold $M$ endowed with an affine flat structure.

By definition, such a structure is given by the subsheaf $\mathcal{T}_{M}^{f} \subset \mathcal{T}_{M}$ of flat vector fields which form a local system of linear spaces and abelian Lie superalgebras (with respect to the supercommutator of vector fields) such that $\mathcal{T}_{M}=\mathcal{O}_{M} \otimes \mathcal{T}_{M}^{f}$. Let $\nabla_{0}: \mathcal{T}_{M} \rightarrow \Omega_{M}^{1} \otimes_{\mathcal{O}_{M}} \mathcal{T}_{M}$ be the associated connection.

Consider a vector field $A$ on $M$. Its covariant differential $\nabla_{0} A$ belongs to $\Omega_{M}^{1} \otimes_{\mathcal{O}_{M}} \mathcal{T}_{M}$. We have the standard isomorphism

$$
j: \Omega_{M}^{1} \otimes \mathcal{O}_{M} \mathcal{T}_{M} \rightarrow \operatorname{End}_{\mathcal{O}_{M}}\left(\mathcal{T}_{M}\right)
$$

Put $B:=j\left(\nabla_{0} A\right)$. We are now in a position to write the commutativity equations for $\left(M, \mathcal{T}_{M}^{f}\right)$ and $B$.

A concrete way of fixing a flat structure consists in giving an atlas with affine linear transition functions between local coordinates of its charts. Such local coordinates $\left(t^{i}\right)$ are then called flat, and $\mathcal{T}_{M}^{f}$ is locally generated by the dual vectors $\partial_{i}=\partial / \partial t^{i}$. When we write the field $B=B(t)$ as a matrix, we always use $\left(\partial_{i}\right)$ as a basis of local sections.

5.3.1. Definition. (i) A solution to the oriented associativity equations is an even vector field $A$ on $M$ with the following property. Write $A=\sum_{c} A^{c} \partial_{c}$ in a local flat coordinate system and put $A_{a b}{ }^{c}:=\partial_{a} \partial_{b} A^{c}$. Then the composition law

$$
\partial_{a} \circ \partial_{b}=\sum_{c} A_{a b}^{c} \partial_{c}
$$


extends to the associative (super)commutative $\mathcal{O}_{M}$-bilinear multiplication on $\mathcal{T}_{M}$.

(ii) An even flat vector field $\partial_{0}$ (contained among $\partial_{i}$ ) is called a flat identity (for $A$ ), if $A_{a 0}{ }^{c}=\delta_{a}^{c}$, or in other words, if $e \circ X=X$ for each vector field $X$.

5.3.2. Proposition. Let $A$ be a solution to the oriented associativity equations with flat identity e. Define $B:=j\left(\nabla_{0} A\right)$ as above and put $h=e$.

Then $B$ is a solution to the commutativity equations with primitive vector $h$.

The map $t \mapsto B(t)$ e establishes a local embedding of $M$ into a fiber of $F$ such that the tautological flat affine structure on $F$ induces the initial affine structure on $M$.

Proof. In the notations of 5.3.1, we have

$$
\begin{gathered}
\nabla_{0} A=\sum_{b, c} d t^{b} \partial_{b} A^{c} \otimes \partial_{c}= \\
=\sum_{b, c}(-1)^{\left(\left|t_{b}\right|+1\right)\left(\left|t_{b}\right|+\left|t_{c}\right|\right)} \partial_{b} A^{c} d t^{b} \otimes \partial_{c} .
\end{gathered}
$$

where $\left|t^{a}\right|$ denotes the parity of $t^{a}$. The last line allows us to compute $j\left(\nabla_{0} A\right)$ as a matrix $B: j\left(d t^{b} \otimes \partial_{c}\right)$ considered as endomorphism of $\mathcal{T}_{M}^{f}$ maps $\partial_{a}$ to $\delta_{a}^{b} \partial_{c}$ so that

$$
B_{b}{ }^{c}=(-1)^{\left(\left|t_{b}\right|+1\right)\left(\left|t_{b}\right|+\left|t_{c}\right|\right)} \partial_{b} A^{c} .
$$

Then a direct calculation shows that the equation $d B \wedge d B=0$ becomes

$$
\forall a, b, c, f, \quad \sum_{e} A_{a b}{ }^{e} A_{e c}^{f}=(-1)^{\left|t^{a}\right|\left(t^{b}|+| t^{c} \mid\right)} \sum_{e} A_{b c}{ }^{e} A_{e a}{ }^{f} .
$$

These are precisely the associativity equations: cf. e. g. [Ma1], pp. 19-20. Since this formulation gives a coordinate free description of the operation $\circ$, it does not depend on the choice of local flat coordinates and agrees on intersections. Supercommutativity of $\circ$ follows from the fact that $\mathcal{T}_{M}^{f}$ is a superabelian Lie algebra so that $A_{a b}{ }^{c}$ is symmetric with respect to its subscripts.

Let now $t=\left(t^{0}, t^{1}, \ldots, t^{m}\right)$ be a flat local coordinate system such that $e=\partial_{0}$. We have $\partial_{a}\left(A_{0}^{c}\right)=\delta_{a}^{c}$, hence $A_{0}^{c}=t^{c}+a^{c}$ where $a^{c}$ are constants. Therefore

$$
B(t) e=\sum_{c}(-1)^{\left|t^{c}\right|}\left(t^{c}+a^{c}\right) \partial_{c} .
$$

This proves the last statement of the Proposition.

The converse statement also holds, at least locally. The point is that for a given $B$, if the equation $B=j\left(\nabla_{0} A\right)$ ) can be solved for $A$ at all, then we can get other solutions by adding to $A$ any flat vector field, so there may be an obstruction for finding a global solution. 
5.3.3. Proposition. Let $B$ be a solution to the commutativity equations for $(M, F)$, and let $h$ be a primitive vector for $B$. Working locally, induce a flat affine structure on $M$ from a fibre $F_{0}$ with the help of the map $t \mapsto B(t) h$.

Then in any local flat coordinate system $\left(t^{c}\right)$ we can define a vector field $\sum_{c} A^{c} \partial_{c}$ such that

$$
\partial_{b} A^{c}=(-1)^{\left(\left|t^{b}\right|+1\right)\left(\left|t^{b}\right|+\left|t^{c}\right|\right)} B_{b}{ }^{c} .
$$

(cf. (5.9)).

This field is a solution to the oriented associativity equations with flat identity $e$ which is the pullback of $h$ (considered as a tangent vector to a fiber of $F$ ) with respect to the map $t \mapsto B(t) h$.

Proof. If we prove the existence of functions $A^{c}$, the rest will follow from the proof of the previous Proposition. The equations for $A^{c}$ are equivalent to

$$
d A^{c}=\sum_{b}(-1)^{\left(\left|t^{b}\right|+1\right)\left(\left|t^{b}\right|+\left|t^{c}\right|\right)} d t^{b} B_{b}^{c}
$$

and their integrability is equivalent to the closedness of all forms

$$
\omega^{c}:=(-1)^{\left(\left|t^{b}\right|+1\right)\left(\left|t^{b}\right|+\left|t^{c}\right|\right)} \sum_{b} d t^{b} B_{b}{ }^{c} .
$$

We will show that the latter in appropriate coordinates is expressed by the equations $d B \wedge d(B h)=0$ which follow from $d B \wedge d B=0$ because $h$ is flat.

In fact, choose a basis of flat vector vector fields of $F_{0}$ containing $h$ and the dual coordinate system $\left(t^{c}\right)$ such that $h=\partial_{0}$. Identifying $M$ (or its local chart) with a subdomain of $F$ via $t \mapsto B(t) h$ we can consider $\left(t^{c}\right)$ as flat coordinates on $M$, and in these coordinates the map $t \mapsto B(t) h$ is given by the formula (5.10). Hence

$$
d(B(t) h)=\sum d t^{b} \otimes \partial_{b}
$$

and

$$
d B \wedge d(B(h))=\sum_{c}\left[\sum_{b}(d B)_{b}{ }^{c} \wedge d t^{b}\right] \otimes \partial_{c}=\sum_{c}(-1)^{\left|t^{c}\right|} d \omega^{c} \otimes \partial_{c}
$$

This completes the proof.

5.4. Formal solutions to the commutativity equations corresponding to $\mathcal{L}$-algebras. Let us now return to the situation described in the Theorem 5.1.1. The formal series $\mathcal{B}$ is a solution to the commutativity equations for the formal manifold $M$ which is the completion of $T \oplus F$ at zero, and trivial local 
system with the fibre $F$. Denote by $\mathcal{T}$ (resp. $\mathcal{F}$ ) formal completions of $T$ (resp. $F$ ) at zero. They are embedded as closed formal submanifolds in $M$.

We can restrict $\mathcal{B}$ to $\mathcal{T}$. Assume that this restriction $\mathcal{B}_{T}$ is maximal. Then in view of the Proposition $5.2 .3, \mathcal{B}$ is a pullback of $\mathcal{B}_{T}$ with respect to a formal projection $\varphi: M \rightarrow \mathcal{T}$.

We can also restrict $\mathcal{B}$ to $\mathcal{F}$. Clearly, $\mathcal{F}$ is endowed with a formal flat structure coming from $F$. The restriction $\mathcal{B}_{F}$ of $\mathcal{B}$ to $\mathcal{F}$ produces a solution to the oriented associativity equations with base $\mathcal{F}$. If the latter admits a primitive vector $h$, we will say that $h$ is weakly primitive for $\mathcal{B}$.

5.4.1. Proposition. Fix two linear superspaces $T$ and $F$ and the following additional data:

(i) A maximal solution $\mathcal{B}_{1}$ to the commutativity equations with the base $\mathcal{T}$ and fiber $F$.

(ii) $A$ solution $\mathcal{B}_{2}$ to the commutativity equations with the base $\mathcal{F}$ and fiber $F$ which comes from a solution to the oriented associativity equations on $\mathcal{F}$ with flat structure induced by $F$, admitting a flat identity $e$.

In this case there exists a pair $(\mathcal{B}, h)$, where $\mathcal{B}$ is a solution to the commutativity equations on the formal completion of $T \oplus F$ at zero, with the fiber $F$, and $h$ is a weakly primitive vector corresponding to e, such that $\mathcal{B}_{1}=\mathcal{B}_{T}, \mathcal{B}_{2}=\mathcal{B}_{F}$. This pair is unique.

Proof. Let $(t)$ denote some coordinates on $\mathcal{T}$ and $(\theta)$ flat coordinates on $\mathcal{F}$. The explicit pullback formula

$$
\mathcal{B}(t, \theta)=\mathcal{B}_{2}\left(\theta+\mathcal{B}_{1}(t) h\right)
$$

produces a solution with necessary properties. It is unique because of maximality of $\mathcal{B}_{1}$.

5.5. Compatibility of two tensor products. Given two structures $I_{i}$ of (oriented) $\mathcal{L}$-algebras upon $\left(T_{i}, F_{i}\right), i=1,2$, we can form the tensor product structure $I=I_{1} \otimes I_{2}$ upon $(T, F)=\left(T_{1} \otimes T_{2}, F_{1} \otimes F_{2}\right)$ imitating the definition 4.2.1 in the nonoriented (cyclic) case. This operation induces the tensor product $*_{C O M M}$ on the formal solutions to the commutativity equations (COMM).

There is also an oriented version $*_{A S S}$ of the tensor product of the formal solutions to the associativity equations ASS (for the cyclic case, see [Ma1], p. 100).

Considering only solutions to ASS with flat identities, and the associated solutions to COMM, we may conjecture that the two operations are compatible. This question was raised in [LoPo] where it was checked that the answer is positive in several first orders. 
Proposition 5.4.1 suggests a strategy for proving this conjecture at least for the case when the solutions to COMM are maximal. However, the most natural and general approach is furnished by the equivalence theorem 5.1.1 and the language of $\mathcal{L}$-algebras where the tensor product is simply induced by the product in the cohomology of moduli spaces, resp. coproduct in their homology. We hope to spell out the details elsewhere.

\section{References}

[BeMa] K. Behrend, Yu. Manin. Stacks of stable maps and Gromov-Witten invariants. Duke Math. Journ., 85:1 (1996), 1-60.

[GeK1] E. Getzler, M. Kapranov. Cyclic operads and cyclic homology. In: Geometry, Topology, and Physics for Raoul, ed. by B. Mazur, Internat. Press, Cambridge, MA, 1995, 167-201.

[GeK2] E. Getzler, M. Kapranov. Modular operads. Compositio Math., 110 (1998), 65-126.

[H] B. Hassett. Moduli spaces of weighted pointed stable curves. Preprint math.AG/0205009

[Ke] S. Keel. Intersection theory of moduli space of stable $N$-pointed curves of genus zero. Trans. AMS, 330:2 (1992), 545-574.

[Kn] Knudsen F.F. The projectivity of the moduli space of stable curves II. The stacks $\bar{M}_{0, n}$. Math. Scand. 52 (1983), 163-199.

[KoMa] M. Kontsevich, Yu. Manin. Gromov-Witten classes, quantum cohomology, and enumerative geometry. Comm. Math. Phys., 164:3 (1994), 525-562.

[KoMaK] M. Kontsevich, Yu. Manin (with Appendix by R. Kaufmann). Quantum cohomology of a product. Inv. Math., 124, f. 1-3 (Remmert's Festschrift) (1996), 313-339.

[Lo1] A.Losev, On "Hodge" Topological Strings at genus zero. Pis'ma v ZhETF 65, 374-379 (1997).

[Lo2] A.Losev, "Hodge strings" and elements of K. Saito's theory of the primitive form. In: Proceedings of Taniguchi Symposium on Topological Field Theory, Primitive Forms and Related Topics, Kyoto, Japan, 9-13 Dec 1996, Springer,1998. Preprint hep-th/9801179

[LoMa] A. Losev, Yu. Manin. New moduli spaces of pointed curves and pencils of flat connections. Michigan Journ. of Math., vol. 48 (Fulton's Festschrift), 2000, 443-472. Preprint math.AG/0001003

[LoPo] A. Losev, I. Polyubin. On compatibility of tensor products on solutions to commutativity and WDVV equations. JETP Letters, 73:2 (2001), 53-58. 
[Ma1] Yu. Manin. Frobenius manifolds, quantum cohomology, and moduli spaces. AMS Colloquium Publications, vol. 47, Providence, RI, 1999, xiii+303 pp.

[Ma2] Yu. Manin. Moduli stacks $\bar{L}_{g, S}$. Preprint math.AG/0206123

[R] M. A. Readdy. The Yuri Manin ring and its $\mathcal{B}_{n}$-analogue. Adv. in Appl. Math., 2001. 